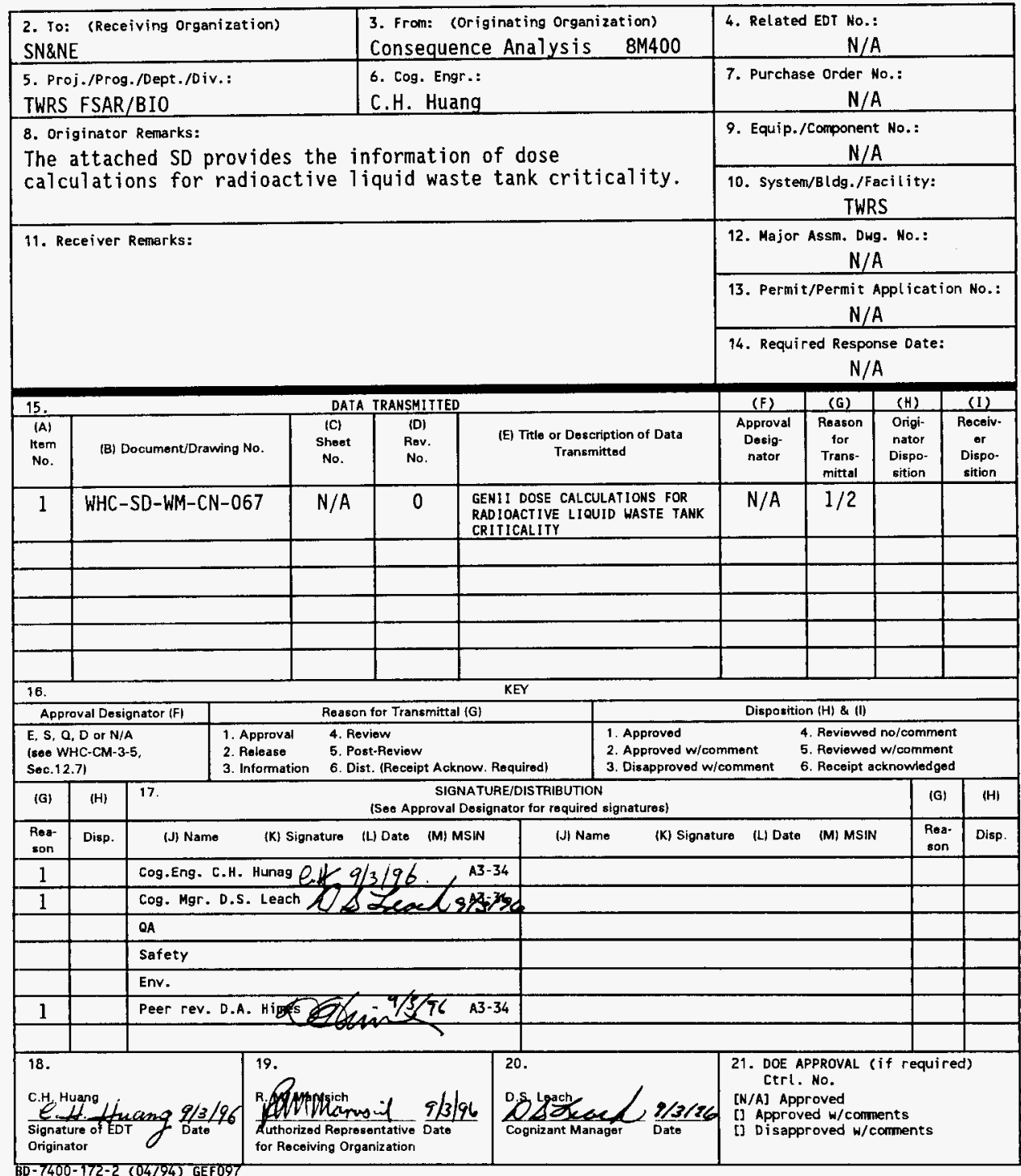




\title{
GENII DOSE CALCULATIONS FOR RADIOACTIVE LIQUID WASTE TANK CRITICALITY
}

\author{
C. H. HUANG
}

Westinghouse Hanford, Richland, WA 99352

U.S. Department of Energy Contract DE-AC06-87RL10930

$\begin{array}{lll}\text { EDT/ECN: } & 156487 & \text { UC: } 2030 \\ \text { Org Code: } & 8 M 400 & \text { Charge Code: NIFC3 } \\ \text { B\&R Code: } & \text { EW3120071 } & \text { Total Pages: } 43\end{array}$

Key Words: Dose Calculation, Liquid Waste Tank, TWRS FSAR, Criticality, Calculation Note.

Abstract: This document provides the values of dose calculations for radioactive liquid waste tank criticality.

TRADEMARK DISCLAIMER. Reference herein to any specific commercial product, process, or service by trade name, trademark, manufacturer, or otherwise, does not necessarily constitute or imply its endorsement, recommendation, or favoring by the United States Goverment or any agency thereof or its contractors or subcontractors.

Printed in the United States of America. To obtain copies of this document, contact: WHC/BCS Document Control Services, P.O. Box 1970, Mailstop H6-08, Richland WA 99352, Phone (509) 372-2420; Fax (509) 376-4989.

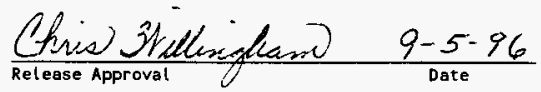

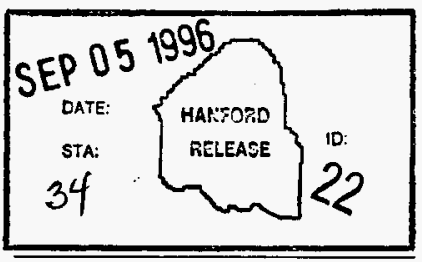

Release Stamp 
WHC-SD-WM-CN-067, Rev. 0

\title{
GENII DOSE CALCULATIONS FOR RADIOACTIVE \\ LIQUID WASTE TANK CRITICALITY
}

\author{
C. H. HUANG
}

August 22, 1996

\section{INTRODUCTION}

The objective for this document is to present the GXQ and GENII calculations for the radioactive liquid waste tank criticality. The release location is at the tank farm in the $200 \mathrm{~W}$ Area.

\section{SOURCE TERM}

GENII code was used to calculate the dose release with the source term for various isotopes specified by the customer as given in Table 1 (see Hey 1992). The assumptions used for evaluation of a criticality are given in the NRC regulatory guides RG. 3.35, the "Assumptions Used for Evaluating the Potential Radiological Consequences of Accidental Nuclear Criticality in a Plutonium Processing and Fuel Fabrication Plant, "(NRC 1979). The Curies content in Table 1 also came from Nuclear Regulatory Commission Regulatory Guide 3.35.

Table 1. Source Term for Various Isotopes.

\begin{tabular}{|l|l|}
\hline Isotope & $\begin{array}{c}\text { Source } \\
\text { Term (Ci) }\end{array}$ \\
\hline KR83M & $1.1 E+02$ \\
\hline KR85M & $7.1 E+01$ \\
\hline KR85 & $8.1 E-04$ \\
\hline KR87 & $4.3 E+02$ \\
\hline KR88 & $2.3 E+02$ \\
\hline KR89 & $1.3 E+04$ \\
\hline XE137M & $1.0 E-01$ \\
\hline$X E 133 M$ & $2.2 E+00$ \\
\hline$X E 133$ & $2.7 E+01$ \\
\hline$X E 135 M$ & $3.3 E+03$ \\
\hline$X E 135$ & $4.1 E+02$ \\
\hline$X E 137$ & $4.9 E+04$ \\
\hline$X E 138$ & $1.1 E+04$ \\
\hline 1131 & $2.8 E+00$ \\
\hline 1132 & $3.0 E+02$ \\
\hline 1133 & $4.0 E+01$ \\
\hline 1134 & $1.1 E+03$ \\
\hline 1135 & $1.1 E+02$ \\
\hline & \\
\hline
\end{tabular}


WHC-SD-WM-CN-067, Rev. O

\section{RECEPTOR LOCATIONS}

The receptor locations are specified by the customer (see Table 2). The receptor locations at the onsite and the offsite boundary for acute ground level releases at the tank farm area are given in Table 2 . The site boundary is near the river bank in the north and east directions.

Table 2. Receptor Locations.

\begin{tabular}{|l|l|}
\hline Receptor & Distance \\
\hline Onsite & $100 \mathrm{~m} \mathrm{E}$ \\
\hline Offsite & $8.76 \mathrm{~km} \mathrm{~N}$ \\
\hline
\end{tabular}

\section{METHODOLOGIES AND ASSUMPTIONS}

The GENII code (Napier et al., 1988) was used to calculate the onsite and offsite doses for inhalation and submersion. The dimension of finite plume was assumed in the GENII calculations. GENII code takes into account the radioactive decay in transit for various isotopes. GXQ code was used to calculate $X / Q$ values for the tank farm project. Since the $X / Q$ values calculated from the GENII code are different from the GXQ code, the doses calculated from the GENII code are adjusted by applying a ratio factor, i.e., the ratio of $X / Q$ value obtained from the GXQ code to that obtained from the GENII code. The heavy metals of Plutonium and Americium are not included in the GENII calculations, which has been reported as a separate issue in another document.

\section{RECEPTOR DESCRIPTIONS}

The onsite and offsite receptor locations were provided by the customer.

- Onsite (Facility): The receptor location is at a distance of $100 \mathrm{~m}$. The receptor at the worst direction is at a distance of $100 \mathrm{~m}$ East.

- Offsite(Site Boundary): Receptor at the site boundary in the worst direction. Where the site is bounded by the Columbia River, the site boundary is taken to be at the near bank of the river at the north and the east directions. The receptor at the worst direction $(8.76 \mathrm{~km} \mathrm{~N})$ was given by the customer.

\section{CODE DOCUMENTATION}

o GXQ version $4.0(11 / 19 / 94)$

- Joint Frequency Data: 200 Area, 10 m, Pasquill A-G (19831991 Average).

o GENII version $1.485(12 / 3 / 90)$ 
WHC-SD-WM-CN-067, Rev. 0

- GENII Default Parameter Values (28-Mar-90 RAP)

o Radionuclide Master Library (7/23/93 PDR)

- External Dose Factor Library (8-May-90-RAP)

- Internal Dose Increments, Maximum Solubilities, (7/23/93 PDR)

GXQ output file and GENII input and output files are attached in Appendix A.

\section{RESULTS}

\section{$X / Q$ CALCULATIONS}

GXQ code (Hey 1994) was used to calculate the $X / Q$ values for the onsite receptor at $100 \mathrm{~m} \mathrm{E}$ and $0 \mathrm{ffsite}$ receptor at $8.76 \mathrm{~km} \mathrm{~N}$. The calculated $X / Q$ values are shown in Table 3 (WHC-SD-WM-SARR-016, Rev. 2).

Table 3. X/Q Values for the Onsite and Offsite Receptors.

\begin{tabular}{|c|c|c|c|c|}
\hline Receptor & Distance & $\begin{array}{c}X / Q\left(\mathrm{~s} / \mathrm{m}^{3}\right) \\
\text { (GXQ cOde) }\end{array}$ & $\begin{array}{c}X / Q\left(\mathrm{~s} / \mathrm{m}^{3}\right) \\
\text { (GENII code) }\end{array}$ & $\begin{array}{c}\text { Ratio } \\
\text { (GXO/GENII) }\end{array}$ \\
\hline Onsite & $100 \mathrm{~m} \mathrm{E}$ & $3.41 \mathrm{E}-02$ & $3.2 \mathrm{E}-02$ & 1.066 \\
\hline Offsite & $8.76 \mathrm{~km} \mathrm{~N}$ & $2.83 \mathrm{E}-05$ & $5.4 \mathrm{E}-05$ & 0.524 \\
\hline
\end{tabular}

\section{DOSE CALCULATIONS}

GENII code was used to calculate the doses with source terms for various isotopes as given in Table 1 . These source terms were used to calculate the doses for onsite and offsite receptors. The calculated doses for inhalation and submersion are given in Table 4.

Table 4. Committed Doses.

\begin{tabular}{||l|c|c|}
\hline Dose Pathway & $\begin{array}{c}\text { Onsite (100 m E) } \\
\mathrm{EDE}(\mathrm{rem})\end{array}$ & $\begin{array}{c}\text { 0ffsite }(8.76 \mathrm{~km} \mathrm{~N}) \\
\mathrm{EDE}(\mathrm{rem})\end{array}$ \\
\hline Inhalation & $7.8 \mathrm{E}+00$ & $8.9 \mathrm{E}-03$ \\
\hline Submersion & $5.1 \mathrm{E}+00$ & $6.8 \mathrm{E}-03$ \\
\hline Total & $1.3 \mathrm{E}+01$ & $1.6 \mathrm{E}-02$ \\
\hline
\end{tabular}




\section{WHC-SD-WM-CN-067, Rev. 0}

The doses calculated from the GENII code were adjusted by a ratio factor shown in Table 3, i.e., the adjusted dose is obtained by multiplying the dose in Table 4 by a ratio factor of 1.066 and 0.524 for the onsite and offsite receptors, respectively. The adjusted doses for the onsite and offsite receptors are shown in Table 5.

Table 5. Committed Doses (Adjusted).

\begin{tabular}{||l|c|c||}
\hline Dose Pathway & $\begin{array}{c}\text { Onsite }(100 \mathrm{~m} \mathrm{E}) \\
\mathrm{EDE}(\mathrm{rem})\end{array}$ & $\begin{array}{c}\text { Offsite }(8.76 \mathrm{~km} \mathrm{~N}) \\
\mathrm{EDE}(\mathrm{rem})\end{array}$ \\
\hline Inhalation & $8.3 \mathrm{E}+00$ & $4.7 \mathrm{E}-03$ \\
\hline Submersion & $5.4 \mathrm{E}+00$ & $3.6 \mathrm{E}-03$ \\
\hline Total & $1.4 \mathrm{E}+01$ & $8.3 \mathrm{E}-02$ \\
\hline
\end{tabular}




\section{WHC-SD-WM-CN-067, Rev, 0}

\section{REFERENCES}

Hey, B. E., 1994, GXQ Program Users' Guide, WHC-SD-GN-SWD-30002, Westinghouse Hanford Company, Richland, Washington.

Napier, B. A., et al., 1988, GENII - The Hanford Environmental Radiation Dosimetry Software System, PNL-6584, Pacific Northwest Laboratory, Richland, Washington.

U.S. Nuclear Regulatory Commission, November 1982, Atmospheric Dispersion Models For Potential Accident Consequence Assessments At Nuclear Power Plants, Regulatory Guide 1.145, Revision 1, Office of Nuclear Regulatory Research.

Van Keuren, J. C., 1996: Tank Waste Compositions and Atmospheric Dispersion Coefficients for Use in Safety Analysis Consequence Assessments, WHCSD-WM-SARR-016, Rev. 2, Westinghouse Hanford Company, Richland, Washington.

NRC, 1979, Assumptions Used for Evaluating the Potential Radiological Consequences of Accidental Nuclear Criticality in a Plutonium Processing and Fuel Fabrication Plant, Regulatory Guides 3.35, U.S. Nuclear Regulatory Commission, Washington, D.C. 
WHC-SD-WM-CN-067, Rev. 0

Appendix A

GXQ OUTPUT FILE AND GENII INPUT AND OUTPUT FILES 
GXQ Version 4.0

Decenber 19, 1994

General Purpose Atmospheric Dispersion Code Produced by West inghouse Hanford Company

Users Guide documented in HHC-SD-GN-SWD-30002 Rev. 1. Validation documented in WHC-SD-GN-SWD-30003 Rev. 1.

Code Custodian is: Brit E. Hey

Westinghouse Hanford Company

P.0. Box 1970

Richland, WA 99352

(509) 376-2921

Run Date $=09 / 02 / 96$

Run Time $=10: 15: 50.96$

INPUT ECHO:

TWRSAR XQs, case 1 . Acute Ground level Releases, no effect c GXQ Version 4.0 input file

c mode

1

c

C MOOE CHOICE:

$c$ mode $=1$ then $X / Q$ based on Hanford site specific meteorology

c mode $=2$ then $X / Q$ based on atmospheric stability class and wind speed

c mode $=3$ then $X / Q$ plot file is created

c

C LOGICAL CHOICES:

c ifox inorm icdf ichk isite ipop

c ifox $=t$ then joint frequency used to compute frequency to exceed $X / Q$

$c \quad=f$ then joint frequency used to compute annual average $x / a$

$c$ inorn $=t$ then joint frequency data is normalized (as in GENII)

$=$ then joint frequency data is un-normal ized

c icdf $=$ t then cumulative distribution file created (CDF.OUT)

c $\quad f$ then no cumulative distribution file created

ichk $=t$ then $X / Q$ parameter print option turned on

$=f$ then no parameter print

isite $=t$ then $X / Q$ based on joint frequency data for all 16 -sectors

$=f$ then $X / Q$ based on joint frequency data of individual sectors

$c$ ipop $=t$ then $x / Q$ is population weighted

$=f$ then no population weighting

C X/Q AND WIND SPEED ADJUSTMENT MOOELS:

ipuff idep isrc iwind

$\begin{array}{llll}0 & 0 & 0 & 0\end{array}$

c DIFFUSION COEFFICIENT ADJUSTMENT MOOELS:

c i wake ipm iflow ientr

0 O 0 O 0

C EFFECTIVE RELEASE HEIGHT ADJUSTMENT MOOELS:

C (irise igrnd)iwash igrav

$$
000
$$

$c$ ipuff $=1$ then $x / Q$ calculated using puff model

c $\quad=0$ then $X / Q$ calculated using default continuous plume model

$c$ idep $=1$ then plume depletion model turned on (Chamberlain model)

$c$ isre $=1$ then $x / 0$ multiplied by scalar

$c \quad=2$ then $X / Q$ adjusted by wind speed function

$c$ iwind $=1$ then wind speed corrected for plume height

$c$ iwake $=1$ then NRC RG 1.145 building wake model turned on

c $\quad=2$ then MACCS virtual distance building wake model turned on

$c$ ipm $=1$ then NRC RG 1.145 plume meander model turned on

$c \quad=2$ then 5 th Power Law plune meander model turned on

$c \quad=3$ then sector average model turned on

$c$ iflow $=1$ then sigmas adjusted for volume flow rate

c ientr $=1$ then method of Pasquill used to account for entrainment

c irise $=1$ then MACCS buoyant plume rise model turned on

$c \quad=2$ then ISC2 momentum/buoyancy plume rise model turned on

$c$ igrnd $=1$ then Mills buoyant plume rise modification for ground effects

$c$ iwash $=1$ then stack downwash model turned on

c igrav = 1 then gravitational settling model turned on

$c \quad=0$ unless specified otherwise, 0 turns model off

c PARAMETER INPUT:

$\begin{array}{lll}\text { c reference } & \text { frequency } \\ \text { c release } & \text { anemometer mixing } & \text { to }\end{array}$ 
WHC-SD-WM-CN-067, Rev. 0

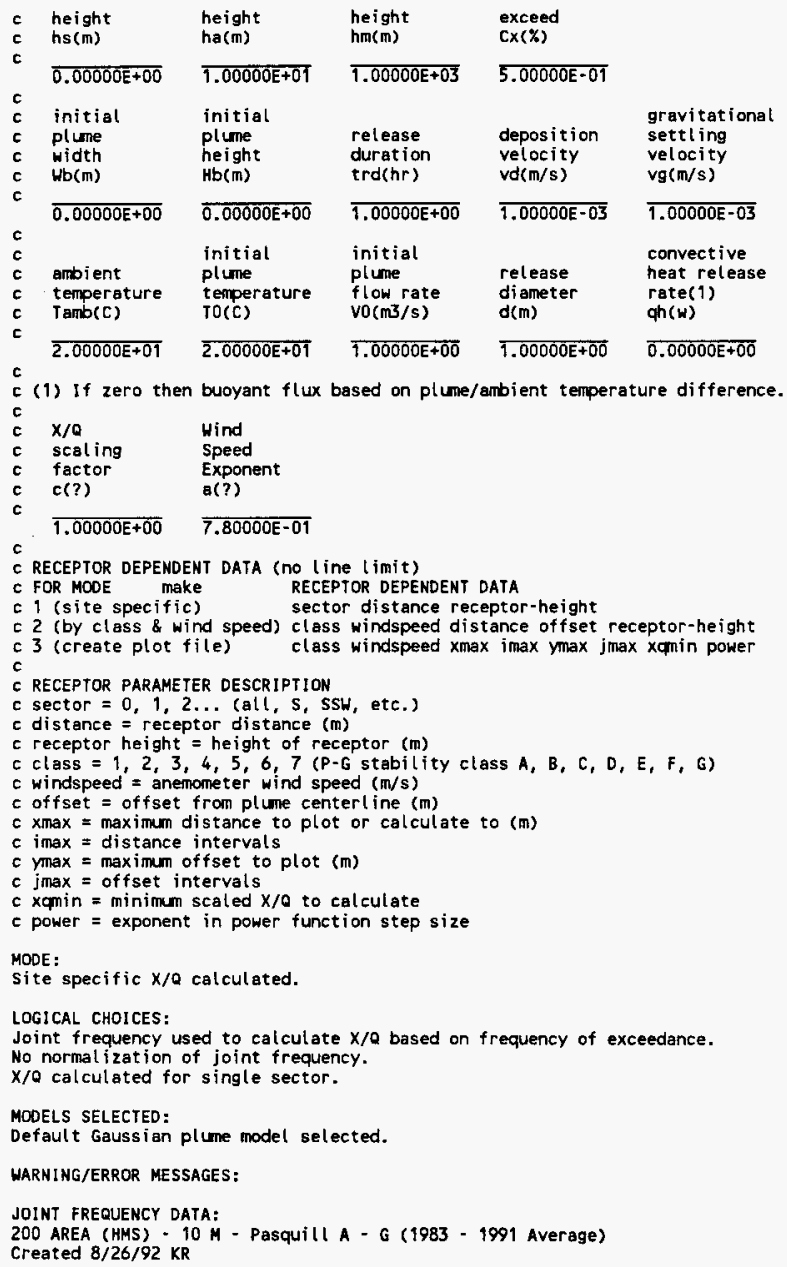


\#\#\#\#\#\#\#\#\#\#\#\#\#\#\#\#\#\#\#\#\#\# Program GENII Input File \#\#\#\#\#\#\#\#\#\#\# 8 Jul 88 \#\#\#\# Title: WASTE TANK CRITICALITY - ONSITE, Inhalation \& Submersion. $\backslash$ GENI I $\backslash$ TANKFC2. IN

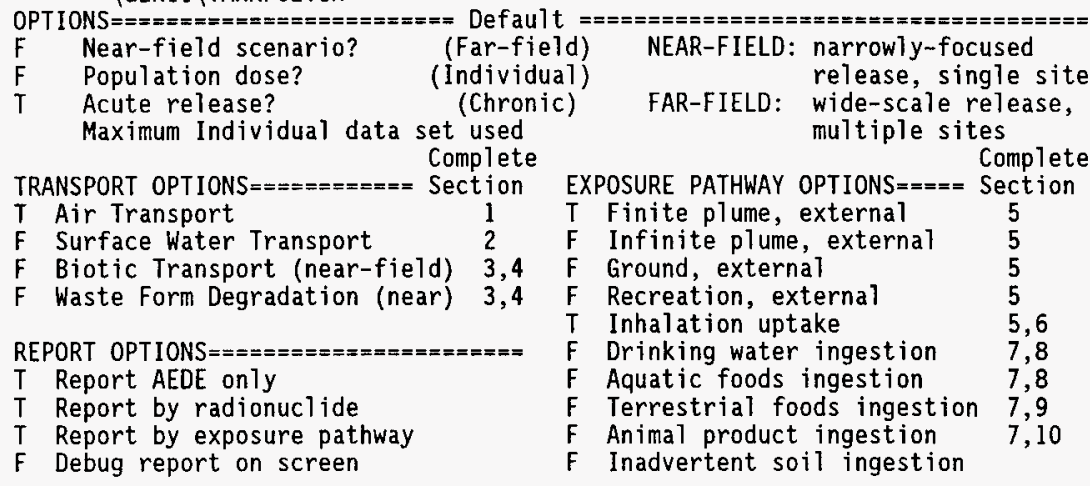

\section{INVENTORY $\# \# \# \# \# \# \# \# \# \# \# \# \# \# \# \# \# \# \# \# \# \# \# \# \# \# \# \# \# \# \# \# \# \# \# \# \# \# \# \# \# \# \# \# \# \# \# \# \# \# \# \# \# \# \# \# \# \# \# \# \# \# \# \# \# \# \# \#$}

4 Inventory input activity units: (1-pCi $2-\mathrm{uCi} 3-\mathrm{mCi} \quad 4-\mathrm{Ci}$ 5-Bq)

0 Surface soil source units (1- m2 2-m3 3- kg)

Equilibrium question goes here

\begin{tabular}{|c|c|c|c|c|c|c|c|c|}
\hline Use when & & ort sel & ected & & field sce & enari & optio & \\
\hline $\begin{array}{l}\text { Release } \\
\text { Radio- } \\
\text { nuclide }\end{array}$ & $\begin{array}{l}\text { Air } \\
/ y r\end{array}$ & $\begin{array}{l}\text { Surface } \\
\text { Water } \\
\text { /yr }\end{array}$ & $\begin{array}{l}\text { Buried } \\
\text { Waste } \\
/ \mathrm{m} 3\end{array}$ & $\begin{array}{l}\text { Air } \\
/ \mathrm{m} 3\end{array}$ & $\begin{array}{l}\text { Surface } \\
\text { Soit } \\
\text { /unit }\end{array}$ & $\begin{array}{l}\text { Deep } \\
\text { Soi1 } \\
/ \mathrm{m}^{3}\end{array}$ & $\begin{array}{l}\text { Ground } \\
\text { Water } \\
/ L\end{array}$ & $\begin{array}{l}\text { Surface } \\
\text { Water } \\
/ L\end{array}$ \\
\hline
\end{tabular}

KR85M 7.1E+01

KR85 8.1E-04

KR87 $\quad 4.3 \mathrm{E}+02$

KR88 2.3E+02

KR89 1.3E+04

XE131M $\quad 1.0 \mathrm{E}-01$

XE133M 2.2E+00

$\begin{array}{ll}X E 133 & 2.7 E+01\end{array}$

XE135M 3.3E+03

$X E 135 \quad 4.1 E+02$

$X E 137 \quad 4.9 E+04$

$X E 138 \quad 1.1 E+04$

I $131 \quad 2.8 \mathrm{E}+00$

I $1323.0 \mathrm{E}+02$

I $1334.0 \mathrm{E}+01$

I $134 \quad 1.1 \mathrm{E}+03$

I $135 \quad 1.1 \mathrm{E}+02$

\begin{tabular}{l|l|l|} 
Use when & measured values are known \\
\hdashline Release & Terres. Animal Drink Aquatic \\
Radio- & Plant Product Water Food
\end{tabular} 
WHC-SD-WM-CN-067, Rev, 0

nuc] ide $\mathrm{l} / \mathrm{kg} \quad / \mathrm{kg} \quad / \mathrm{L} \quad / \mathrm{kg} /$

TIME \#\#\#\#\#\#\#\#\#\#\#\#\#\#\#\#\#\#\#\#\#\#\#\#\#\#\#\#\#\#\#\#\#\#\#\#\#\#\#\#\#\#\#\#\#\#\#\#\#\#\#\#\#\#\#\#\#\#\#\#\#\#\#\#\#\#\#\#\#\#\#\#\#

$1 \quad$ Intake ends after (yr)

50 Dose calc. ends after $(y r)$

0 Release ends after (yr)

0 No. of years of air deposition prior to the intake period

0 No. of years of irrigation water deposition prior to the intake period

FAR-FIELD SCENARIOS (IF POPULATION DOSE) \#\#\#\#\#\#\#\#\#\#\#\#\#\#\#\#\#\#\#\#\#\#\#\#\#\#\#\#\#\#\#\#\#

0 Definition option: 1-Use population grid in file POP. IN

0 2-Use total entered on this line

\section{NEAR-FIELD SCENARIOS \#\#\#\#\#\#\#\#\#\#\#\#\#\#\#\#\#\#\#\#\#\#\#\#\#\#\#\#\#\#\#\#\#\#\#\#\#\#\#\#\#\#\#\#\#\#\#\#\#\#\#\#\#\#\#\#\#}

Prior to the beginning of the intake period: (yr)

0 When was the inventory disposed? (Package degradation starts)

$0 \quad$ When was LOIC? (Biotic transport starts)

$0 \quad$ Fraction of roots in upper soil (top $15 \mathrm{~cm}$ )

$0 \quad$ Fraction of roots in deep soil

0 Manual redistribution: deep soil/surface soil dilution factor

0 Source area for external dose modification factor (m2)

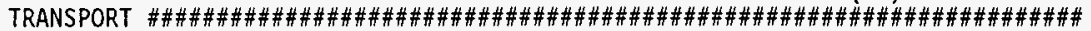

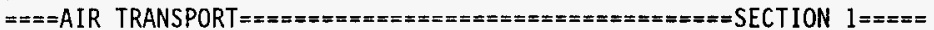

0 -Calculate $P M$

Option: 1-Use chi/Q or PM value 2-Select MI dist \& dir 3-Specify MI dist \& dir

0

13

100 .

$\mathrm{T}$

0

0

0

0

0

0

0

0

0

0

0
0
0

$T$

$T$

0
Chi $/ Q$ or $P M$ value
MI sector index $(l=S)$

MI distance from release point (m) 0

Use jf data, (T/F) else chi/Q gridio
Mixing ratio, dimensionless

If mixing ratio model $>0$ :

Waste form/package half life, (yr)

Waste thickness, (m)

Depth of soil overburden, $m$
Release type (0-3)

Stack release (T/F)

Stack height (m)

Stack flow $(\mathrm{m} 3 / \mathrm{sec})$

Stack radius ( $\mathrm{m}$ )

Effluent temp. (C)

Building $x$-section ( $m 2$ )

Building height (m)

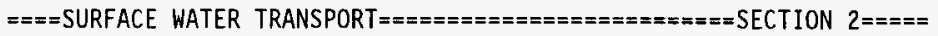
Mixing ratio mode1: 0-use value, 1-river, 2-1ake

Average river flow rate for: MIXFLG $=0(\mathrm{~m} 3 / \mathrm{s}), \operatorname{MIXFLG}=1,2(\mathrm{~m} / \mathrm{s})$, Transit time to irrigation withdrawl location (hr)

Rate of effluent discharge to receiving water body (m3/s)

Longshore distance from release point to usage location (m)

offshore distance to the water intake (m)

Average water depth in surface water body $(\mathrm{m})$

Average river width (m), MIXFLG=1 only

Depth of effluent discharge point to surface water (m), lake only

$====$ WASTE FORM AVAILABILITY=m=s=m

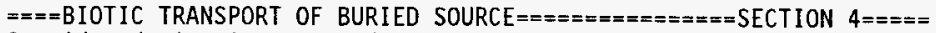
Consider during inventory decay/buildup period (T/F)?

Consider during intake period (T/F)? | 1-Arid non agricultural

Pre-Intake site condition............ 2-Humid non agricultural 
WHC-SD-WM-CN-067, Rev. 0

3-Agricul tural

EXPOSURE \#\#\#\#\#\#\#\#\#\#\#\#\#\#\#\#\#\#\#\#\#\#\#\#\#\#\#\#\#\#\#\#\#\#\#\#\#\#\#\#\#\#\#\#\#\#\#\#\#\#\#\#\#\#\#\#\#\#\#\#\#\#\#\#\#\#\#\#\#

0

0

0

0

0

0

1.0

8766.0

0

0

0

0

0

0

F

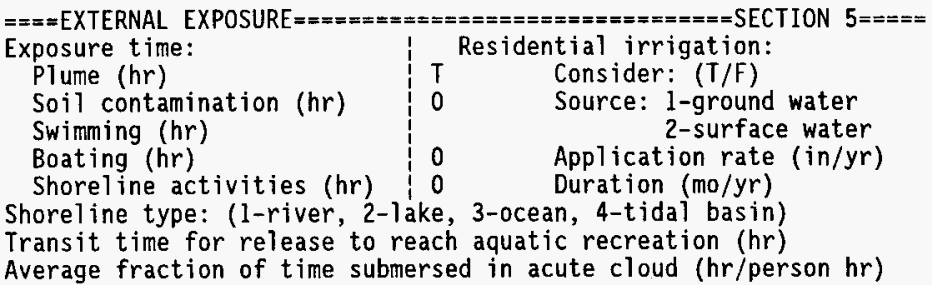

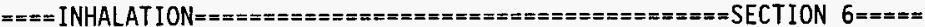
Hours of exposure to contamination per year

0-No resus- 1-Use Mass Loading 2-Use Anspaugh mode1 pension Mass loading factor $(\mathrm{g} / \mathrm{m} 3)$ Top soil available $(\mathrm{cm})$

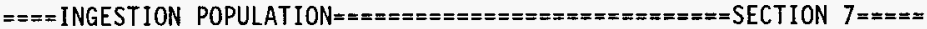
Atmospheric production definition (select option):

0 -Use food-weighted $\operatorname{chi} / Q,($ food-sec $/ \mathrm{m} 3)$, enter value on this line 1-Use population-weighted chi/Q

2-Use uniform production

3-Use chi/Q and production grids (PRODUCTION will be overridden) Population ingesting aquatic foods, 0 defaults to total (person)

Population ingesting drinking water, 0 defaults to total (person)

Consider dose from food exported out of region (default $t=F$ )

Note below: $5^{*}$ or Source: 0 -none, 1-ground water, 2-surface water 3-Derived concentration entered above $====$ AQUATIC FOODS / DRINKING WATER INGESTION $========$ SECTION $8====$

Salt water? (default is fresh)

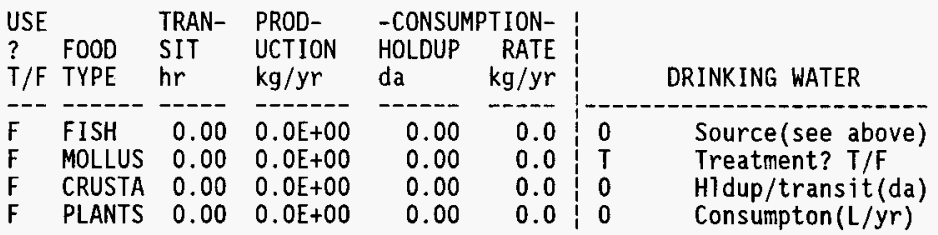

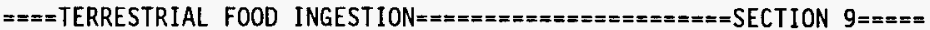

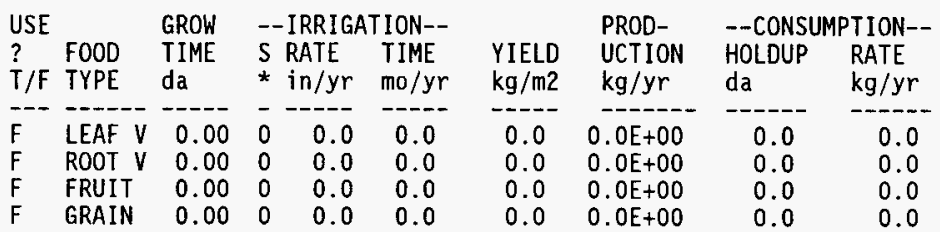

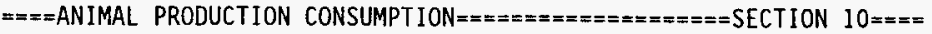


WHC-SD-WM-CN-067, Rev. 0

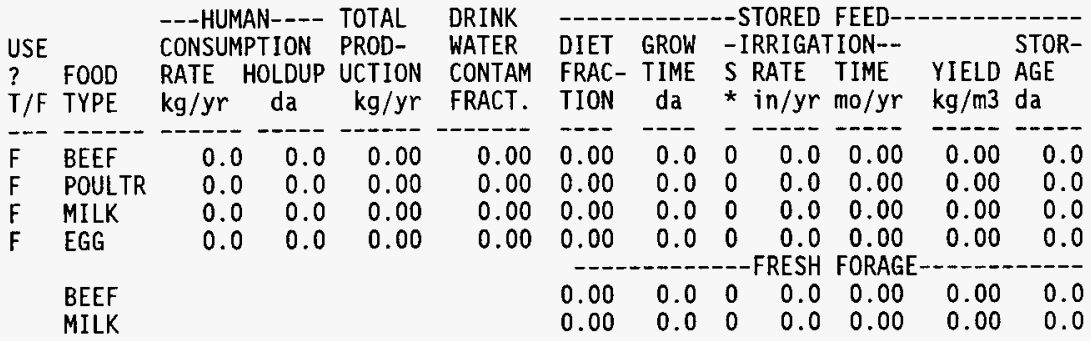

\#\#\#\#\#\#\#\#\#\#\#\#\#\#\#\#\#\#\#\#\#\#\#\#\#\#\#\#\#\#\#\#\#\#\#\#\#\#\#\#\#\#\#\#\#\#\#\#\#\#\#\#\#\#\#\#\#\#\#\#\#\#\#\#\#\#\#\#\#\#\#\#\#\#\#\#\# 
\#\#\#\#\#\#\#\#\#\#\#\#\#\#\#\#\#\#\#\#\#\# Program GENII Input File \#\#\#\#\#\#\#\#\#\#\#8 Jul 88 \#\#\#\# Title: WASTE TANK CRITICALITY - OFFSITE, Inhalation \& Submersion. $\backslash$ GENI I $\backslash T A N K F F C 2$. IN

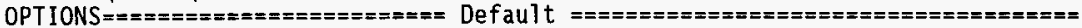
$\begin{array}{llll}F & \text { Near-field scenario? } & \text { (Far-field) } & \text { NEAR-FIELD: narrowly-focused } \\ F & \text { Population dose? } & \text { (Individual) } & \\ \text { T } & \text { Acute release? } & \text { (Chronic) } & \text { FAR-FIELD: wide-scale release, }\end{array}$ Maximum Individual data set used Complete TRANSPORT OPTIONS======s=m=== Sect ion

T Air Transport

F Surface Water Transport

F Biotic Transport (near-field) 3,4

$F$ Waste Form Degradation (near) 3,4

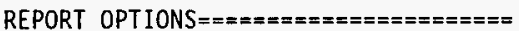

$T$ Report AEDE only

$T$ Report by radionuclide

$T$ Report by exposure pathway

$F$ Debug report on screen multiple sites

Complete

EXPOSURE PATHWAY OPTIONS $=====$ Section

INVENTORY \#\#\#\#\#\#\#\#\#\#\#\#\#\#\#\#\#\#\#\#\#\#\#\#\#\#\#\#\#\#\#\#\#\#\#\#\#\#\#\#\#\#\#\#\#\#\#\#\#\#\#\#\#\#\#\#\#\#\#\#\#\#\#\#\#\#

4 Inventory input activity units: (1-pCi $2-u C i \quad 3-m C i \quad 4-C i \quad 5-B q)$

0 Surface soil source units $\left(1-\mathrm{m} 22-\mathrm{m}^{3} 3-\mathrm{kg}\right)$

Equilibrium question goes here

\begin{tabular}{|c|c|c|c|c|c|c|c|c|}
\hline Use when & & ort sel & ected & & field & enar & opt & ly \\
\hline $\begin{array}{l}\text { Release } \\
\text { Radio- } \\
\text { nuclide }\end{array}$ & $\begin{array}{l}\text { Air } \\
\text { /yr }\end{array}$ & $\begin{array}{l}\text { Surface } \\
\text { Water } \\
\text { /yr }\end{array}$ & $\begin{array}{l}\text { Buried } \\
\text { Waste } \\
\text { /m3 }\end{array}$ & $\begin{array}{l}\text { Air } \\
/ \mathrm{m} 3\end{array}$ & $\begin{array}{l}\text { Surface } \\
\text { Soil } \\
\text { /unit }\end{array}$ & $\begin{array}{l}\text { Deep } \\
\text { Soil } \\
/ \mathrm{m} 3\end{array}$ & $\begin{array}{l}\text { Ground } \\
\text { Water } \\
/ \mathrm{L}\end{array}$ & $\begin{array}{l}\text { Surface } \\
\text { Water } \\
/ L\end{array}$ \\
\hline
\end{tabular}

KR85M 7.1E+01

KR85 8.1E-04

KR87 4.3E+02

KR88 $\quad 2.3 \mathrm{E}+02$

KR89 1.3E+04

XE131M 1.0E-01

XE133M 2.2E+00

$X E 133 \quad 2.7 E+01$

$X E 135 M \quad 3.3 E+03$

$X E 135 \quad 4.1 E+02$

$X E 137 \quad 4.9 E+04$

$X E 138 \quad 1.1 \mathrm{E}+04$

I $131 \quad 2.8 \mathrm{E}+00$

I $132 \quad 3.0 E+02$

I $1334.0 \mathrm{E}+01$

I $134 \quad 1.1 \mathrm{E}+03$

I $135 \quad 1.1 \mathrm{E}+02$

\begin{tabular}{l|l|} 
Use when & \multicolumn{1}{c}{ measured values are known } \\
\hline Release & Terres. Animal Drink Aquatic \\
Radio- & Plant Product Water Food
\end{tabular} 
WHC-SD-WM-CN-067, Rev. 0

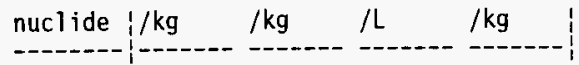

TIME \#\#\#\#\#\#\#\#\#\#\#\#\#\#\#\#\#\#\#\#\#\#\#\#\#\#\#\#\#\#\#\#\#\#\#\#\#\#\#\#\#\#\#\#\#\#\#\#\#\#\#\#\#\#\#\#\#\#\#\#\#\#\#\#\#\#\#\#\#\#\#\#\#

$1 \quad$ Intake ends after (yr)

50 Dose calc. ends after $(y r)$

0 Release ends after (yr)

0 No. of years of air deposition prior to the intake period

0 No. of years of irrigation water deposition prior to the intake period

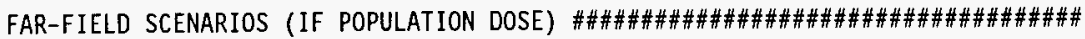

0 Definition option: 1-Use population grid in file POP.IN

0

2-Use total entered on this line

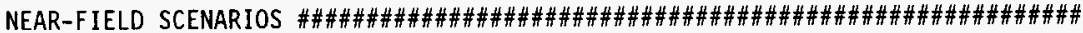

Prior to the beginning of the intake period: ( $y r)$

0

0

0

0

0

0

TRANSPORT

3

0

9 8760.

When was the inventory disposed? (Package degradation starts) When was LOIC? (Biotic transport starts)

Fraction of roots in upper soil (top $15 \mathrm{~cm}$ )

Fraction of roots in deep soil

Manual redistribution: deep soil/surface soil dilution factor Source area for external dose modification factor (m2)

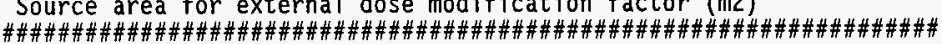

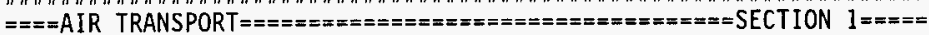
\begin{tabular}{c|cc}
0 -Calculate PM & Release type $(0-3)$
\end{tabular}

Option: 1-Use chi/Q or PM value iF Stack release (T/F) 2-Select MI dist \& dir 3-Specify MI dist \& dir

0

0

0 Chi/Q or PM value

MI sector index $(\mathrm{I}=\mathrm{S})$

0

MI distance from release point $(\mathrm{m})$ Use jf data, (T/F) else chi/Q gridio

Stack height $(\mathrm{m})$

Stack flow $(\mathrm{m} 3 / \mathrm{sec})$

Stack radius (m)

Effluent temp. (C)

Building $x$-section ( $m 2)$

Building height $(\mathrm{m})$

$====$ SURFACE WATER TRANSPORT $==== \pm==================$ SECTION $2=====$ Mixing ratio model: 0-use value, 1-river, 2-lake

Mixing ratio, dimensionless

Average river flow rate for: MIXFLG $=0(\mathrm{~m} 3 / \mathrm{s})$, MIXFLG $=1,2(\mathrm{~m} / \mathrm{s})$, Transit time to irrigation withdrawl location (hr)

If mixing ratio model $>0$ :

Rate of effluent discharge to receiving water body $(\mathrm{m} 3 / \mathrm{s})$

Longshore distance from release point to usage location (m)

Offshore distance to the water intake (m)

Average water depth in surface water body $(\mathrm{m})$

Average river width (m), MIXFLG $=1$ only

Depth of effluent discharge point to surface water $(\mathrm{m})$, lake only

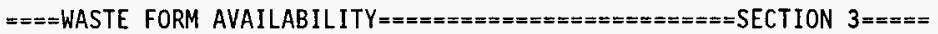
Waste form/package half life, (yr)

Waste thickness, (m)

Depth of soil overburden, $m$

$===$ BIOTIC TRANSPORT OF BURIED SOURCE $=============$ SECTION $4====$ Consider during inventory decay/buildup period (T/F)?

Consider during intake period (T/F)? l-Arid non agricultural

Pre-Intake site condition.............. 2-Humid non agricultural 


\section{3-Agricultural}

EXPOSURE $\# \# \# \# \# \# \# \# \# \# \# \# \# \# \# \# \# \# \# \# \# \# \# \# \# \# \# \# \# \# \# \# \# \# \# \# \# \# \# \# \# \# \# \# \# \# \# \# \# \# \# \# \# \# \# \# \# \# \# \# \# \# \# \# \# \# \# \# \#$

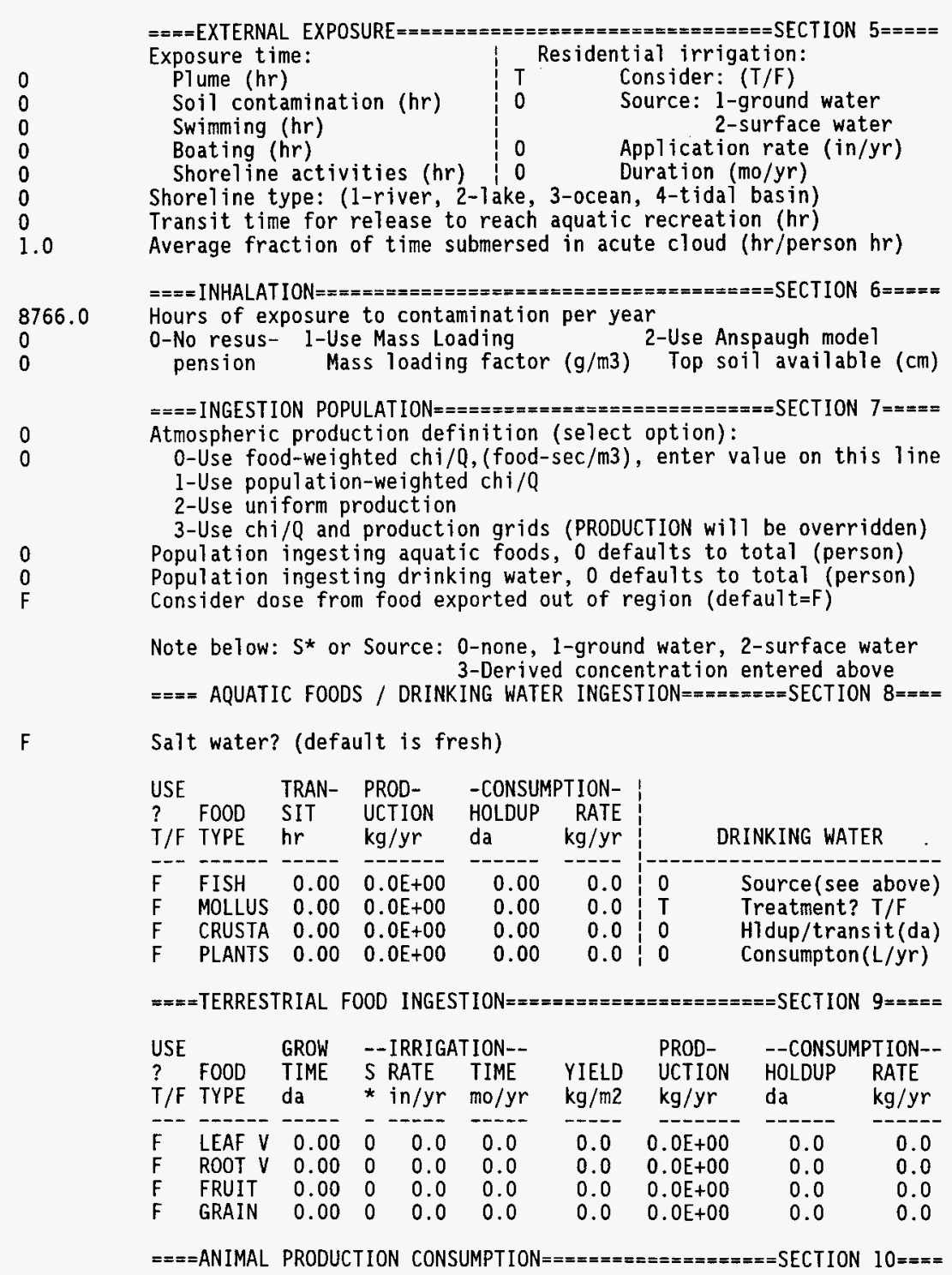


WHC-SD-WM-CN-067, Rev. 0

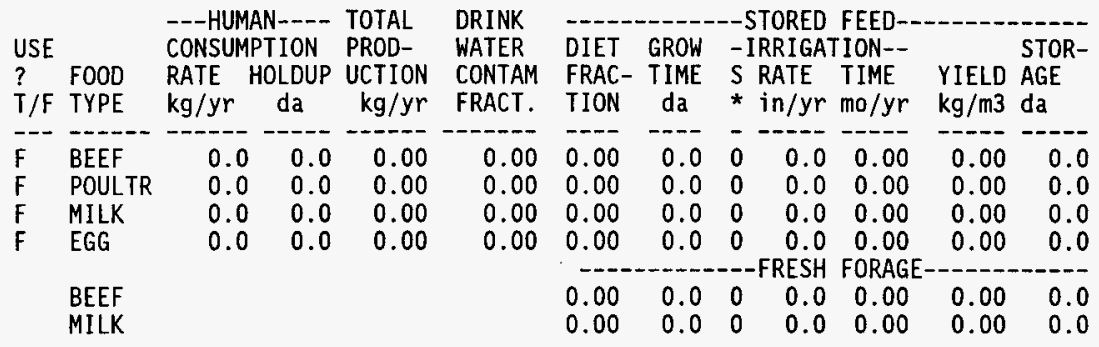

\#\#\#\#\#\#\#\#\#\#\#\#\#\#\#\#\#\#\#\#\#\#\#\#\#\#\#\#\#\#\#\#\#\#\#\#\#\#\#\#\#\#\#\#\#\#\#\#\#\#\#\#\#\#\#\#\#\#\#\#\#\#\#\#\#\#\#\#\#\#\#\#\#\#\#\#\# 
(Version 1.485 3-Dec-90)

Case title: WASTE TANK CRITICALITY - ONSITE, Inhalation \& Submersion. Executed on: $08 / 13 / 96$ at $15: 01: 52$

Page A. 1

This is a far-field (wide-scale release, multiple site) scenario.

Release is acute

Individual dose

THE FOLLOWING TRANSPORT MODES ARE CONSIDERED

Air

THE FOLLOWING EXPOSURE PATHS ARE CONSIDERED:

Finite plume, external

Inhalation uptake

THE FOLLOWING TIMES ARE USED:

Intake ends after $(y r)$ : $\quad 1.0$

Dose calculations ends after (yr): $\quad 50.0$

$========+=$ FILENAMES AND TITLES OF FILES/LIBRARIES USED

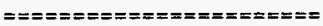

Input file name: \GENII\TANKFC2. IN

GENII Default Parameter Values (28-Mar-90 RAP)

Radionuclide Library - Times $<100$ years (23-July-93 PDR)

External Dose Factors for GENII in person Sv/yr per Bq/n (8-May-90 $R$

Worst-Case Solubilities, Yearly Dose Increments (23-Jul-93 PDR)

EXTGAM - Gamma Energies by Group for Finite Plume (13-May-90 RAP)

200 AREA (HMS) - 10 M - Pasquil1 A - G (1983 - 1991 Average)

\begin{tabular}{|c|c|c|c|}
\hline $\begin{array}{l}\text { Release } \\
\text { Radio- } \\
\text { nuclide }\end{array}$ & $\begin{array}{l}\text { Air } \\
\mathrm{Ci} / \mathrm{yr}\end{array}$ & $\begin{array}{c}\text { Surface } \\
\text { Water } \\
\mathrm{Ci} / \mathrm{yr}\end{array}$ & $\begin{array}{r}\text { Buried } \\
\text { Source } \\
\mathrm{Ci} / \mathrm{m} 3\end{array}$ \\
\hline $\begin{array}{l}\text { KR83M } \\
\text { KR85M } \\
\text { KR85 } \\
\text { KR87 } \\
\text { KR88 }\end{array}$ & $\begin{array}{l}1.1 \mathrm{E}+02 \\
7.1 \mathrm{E}+01 \\
8.1 \mathrm{E}-04 \\
4.3 \mathrm{E}+02 \\
2.3 \mathrm{E}+02\end{array}$ & $\begin{array}{l}0.0 \mathrm{E}+00 \\
0.0 \mathrm{E}+00 \\
0.0 \mathrm{E}+00 \\
0.0 \mathrm{E}+00 \\
0.0 \mathrm{E}+00\end{array}$ & $\begin{array}{l}0.0 \mathrm{E}+00 \\
0.0 \mathrm{E}+00 \\
0.0 \mathrm{E}+00 \\
0.0 \mathrm{E}+00 \\
0.0 \mathrm{E}+00\end{array}$ \\
\hline
\end{tabular}


WHC-SD-WM-CN-067, Rev. 0

$\begin{array}{llll}\text { KR89 } & 1.3 \mathrm{E}+04 & 0.0 \mathrm{E}+00 & 0.0 \mathrm{E}+00 \\ \text { XE131M } & 1.0 \mathrm{E}-01 & 0.0 \mathrm{E}+00 & 0.0 \mathrm{E}+00 \\ \text { XE133M } & 2.2 \mathrm{E}+00 & 0.0 \mathrm{E}+00 & 0.0 \mathrm{E}+00 \\ \text { XE133 } & 2.7 \mathrm{E}+01 & 0.0 \mathrm{E}+00 & 0.0 \mathrm{E}+00 \\ \text { XE135M } & 3.3 \mathrm{E}+03 & 0.0 \mathrm{E}+00 & 0.0 \mathrm{E}+00 \\ \text { XE135 } & 4.1 \mathrm{E}+02 & 0.0 \mathrm{E}+00 & 0.0 \mathrm{E}+00 \\ \text { XE137 } & 4.9 \mathrm{E}+04 & 0.0 \mathrm{E}+00 & 0.0 \mathrm{E}+00 \\ \text { XE138 } & 1.1 \mathrm{E}+04 & 0.0 \mathrm{E}+00 & 0.0 \mathrm{E}+00 \\ \mathrm{I} 131 & 2.8 \mathrm{E}+00 & 0.0 \mathrm{E}+00 & 0.0 \mathrm{E}+00 \\ \mathrm{I} 132 & 3.0 \mathrm{E}+02 & 0.0 \mathrm{E}+00 & 0.0 \mathrm{E}+00 \\ \text { I } 133 & 4.0 \mathrm{E}+01 & 0.0 \mathrm{E}+00 & 0.0 \mathrm{E}+00 \\ \text { I } 134 & 1.1 \mathrm{E}+03 & 0.0 \mathrm{E}+00 & 0.0 \mathrm{E}+00 \\ \mathrm{I} 135 & 1.1 \mathrm{E}+02 & 0.0 \mathrm{E}+00 & 0.0 \mathrm{E}+00\end{array}$

$= \pm= \pm== \pm= \pm=$ AIR TRANSPORT

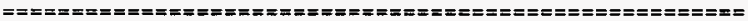
Joint frequency data input.

$1.0 \mathrm{E}+02$ Maximum individual distance from release point (m)

1.3E +01 Maximum individual sector index (Wind Toward E) Ground level release.

$===s======$ EXTERNAL EXPOSURE

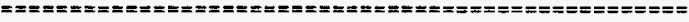

$1.0 \mathrm{E}+00$ Fraction of time spent in cloud

$====x====0$ INHALATION

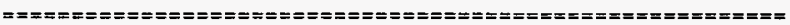

Resuspension not considered

Input prepared by:

Date:

Input checked by:

Date: 
WHC-SD-WM-CN-067, Rev. 0

\title{
GENII Dose Calculation Program
}

(Version 1.485 3-Dec-90)

\begin{abstract}
Case title: WASTE TANK CRITICALITY - ONSITE, Inhalation \& Submersion.
Executed on: $08 / 13 / 96$ at 15:01:52

Page A.
\end{abstract}

Sector Index: 13

Probability: 5.0E-02

\begin{tabular}{|c|c|c|c|c|c|c|c|}
\hline $\begin{array}{l}\text { Distance } \\
\text { (m) }\end{array}$ & $\begin{array}{l}\text { Travel } \\
\text { Time } \\
\text { (sec) }\end{array}$ & $\begin{array}{l}\text { Energy } \\
0.15 \\
(\mathrm{MeV})\end{array}$ & $\begin{array}{l}\text { Energy } \\
0.4 \\
(\mathrm{MeV})\end{array}$ & $\begin{array}{l}\text { Energy } \\
0.75 \\
(\mathrm{MeV})\end{array}$ & $\begin{array}{l}\text { Energy } \\
1.25 \\
(\mathrm{MeV})\end{array}$ & $\begin{array}{l}\text { Energy } \\
1.75 \\
(\mathrm{MeV})\end{array}$ & $\begin{array}{l}\text { Energy } \\
2.25 \\
(\mathrm{MeV})\end{array}$ \\
\hline 100. & 38. & $1.6 \mathrm{E}-04$ & $1.7 \mathrm{E}-04$ & $1.7 \mathrm{E}-04$ & 1. $6 \mathrm{E}-04$ & $1.4 \mathrm{E}-04$ & 1. $2 E-04$ \\
\hline
\end{tabular}


WHC-SD-WM-CN-067, Rev. 0

GENII Dose Calculation Program

(Version 1.485 3-Dec-90)

Case title: WASTE TANK CRITICALITY - ONSITE, Inhalation \& Submersion.

Executed on: $08 / 13 / 96$ at $15: 02: 00$

Page B. 1

3.2E-02 Individual $\mathrm{E} / \mathrm{Q}$ 
WHC-SD-WM-CN-067, Rev. 0

GENII Dose Calculation Program

(Version 1.485 3-Dec-90)

Case title: WASTE TANK CRITICALITY - ONSITE, Inhalation \& Submersion. 1

Executed on: $08 / 13 / 96$ at $15: 02: 03$

Page $C$.

Acute release

Uptake/exposure period:

Dose commitment period:

Dose units:

$\operatorname{Rem}^{1.0} \begin{array}{r}50.0 \\ \end{array}$

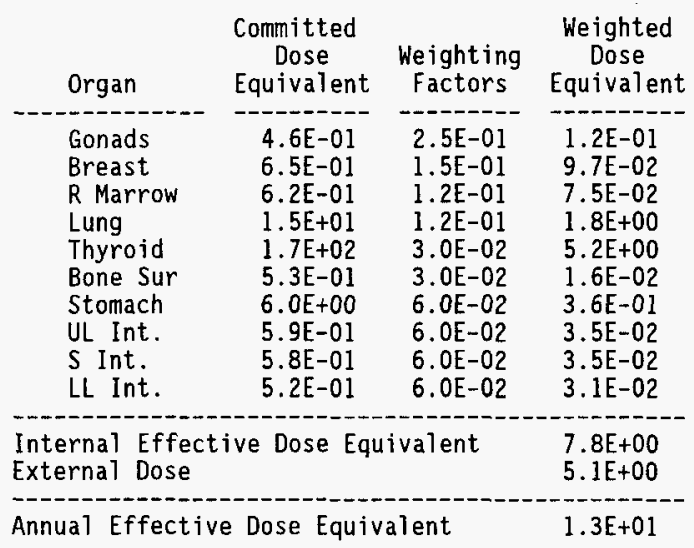

\begin{tabular}{ll} 
Controlling Organ: & Thyroid \\
Controlling Pathway: & Inh \\
Controlling Radionuclide: & I 133 \\
\hline Total Inhalation EDE: & $7.8 \mathrm{E}+00$ \\
Total Ingestion EDE: & $0.0 \mathrm{E}+00$
\end{tabular}


WHC-SD-WM-CN-067, Rev. 0

GENII Dose Calculation Program

(Version 1.485 3-Dec-90)

Case title: WASTE TANK CRITICALITY - ONSITE, Inhalation \& Submersion.

Executed on: $08 / 13 / 96$ at $15: 02: 03$

Page $C$. 2

Acute release

Uptake/exposure period:

Dose commitment period:

Dose units:

1.0

50.0

Rem

Dose Commitment Year

$\begin{array}{llll}1 & 2 & 3 & \ldots\end{array}$

Internal : :

Intake : :

Year: $3: 0.0 \mathrm{E}+00$

$1+$

$2: 0.0 \mathrm{E}+00 \quad 0.0 \mathrm{E}+00 \quad \ldots \quad$ Internal

$+++\quad$ Effective

$1: 7.8 E+00+3.7 E-05+3.8 E-06+\ldots=7.8 E+00$ Dose

Equivalent

i! $\quad$ i 11

Internal

Cumulative

Annual

$7.8 \mathrm{E}+00+3.7 \mathrm{E}-05+3.8 \mathrm{E}-06+\ldots=7.8 \mathrm{E}+00$

Internal

Dose

Dose

$+\quad+\quad+$

External

$\begin{array}{llllll}\text { Annual } & 5.1 \mathrm{E}+00 & 0.0 \mathrm{E}+00 & 0.0 \mathrm{E}+00 & \ldots & 5.1 \mathrm{E}+00\end{array}$

Dose 
WHC-SD-WM-CN-067, Rev. 0

II $\quad$ it $\quad$ it

Annual

Cumulative

Dose

$1.3 \mathrm{E}+01+3.7 \mathrm{E}-05+3.8 \mathrm{E}-06+\ldots$

$=1.3 \mathrm{E}+01$

Dose

Maximum

1.3E+01 Annual

Dose Occurred

In Year 1 
WHC-SD-WM-CN-067, Rev. 0

GENII Dose Calculation Program

(Version 1.485 3-Dec-90)

Case title: WASTE TANK CRITICALITY - ONSITE, Inhalation \& Submersion.

Executed on: $08 / 13 / 96$ at $15: 02: 03$

Page C. 3

Acute release

Uptake/exposure period:

Dose commitment period:

Dose units:

$$
\text { Rem } \begin{array}{r}
1.0 \\
50.0
\end{array}
$$

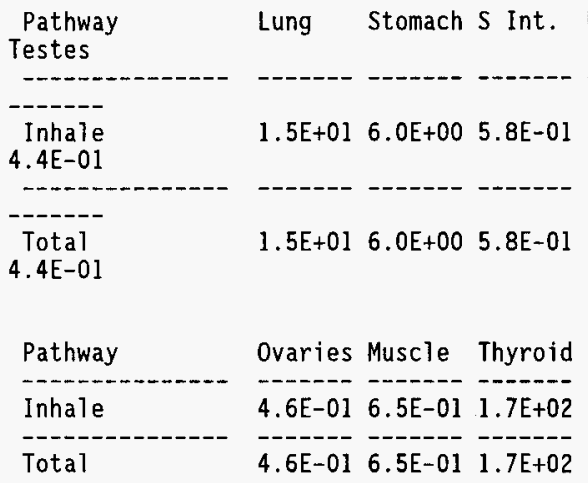

External Dose by Exposure Pathway

Pathway

\begin{tabular}{ll} 
Plume & $5.1 \mathrm{E}+00$ \\
\hline Total & $5.1 \mathrm{E}+00$
\end{tabular}


(Version 1.485 3-Dec-90)

Case title: WASTE TANK CRITICALITY - ONSITE, Inhalation \& Submersion.

Executed on: $08 / 13 / 96$ at $15: 02: 03$

Page $C$. 4

Acute release

Uptake/exposure period:

Dose commitment period:

Dose units:

$$
\text { Rem } \begin{array}{r}
1.0 \\
50.0
\end{array}
$$

Committed Dose Equivalent by Radionuclide

Radionuclide Lung Stomach S Int. UL Int. LL Int. Bone Su R Marro Testes

KR $83 M$
$0.0 E+00$
$K R 85 M$
$0.0 E+00$
$K R 85$
$0.0 E+00$
$K R 87$
$0.0 E+00$
$R B 87$
$2.1 E-16$
$K R 88$
$0.0 E+00$
$R B 88$
$2.8 E-04$
$K R 89$
$0.0 E+00$
$R B 89$
$1.7 E-02$
$S R 89$
$8.5 E-06$
$I 131$
$2.4 E-03$
$X E 131 M$
$0.0 E+00$
$I 132$
$1.2 E-01$
$I \quad 133$
$3.2 E-02$
$X E 133 M$
$0.0 E+00$
$X E 133$
$0.0 E+00$
$I 134$
$1.9 E-01$

$\begin{array}{lllllll}0.0 E+00 & 0.0 E+00 & 0.0 E+00 & 0.0 E+00 & 0.0 E+00 & 0.0 E+00 & 0.0 E+00\end{array}$

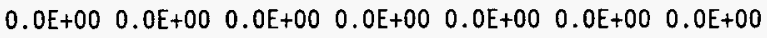
$\begin{array}{llllllllll}0.0 E+00 & 0.0 E+00 & 0.0 E+00 & 0.0 E+00 & 0.0 E+00 & 0.0 E+00 & 0.0 E+00\end{array}$

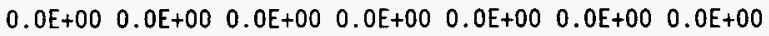

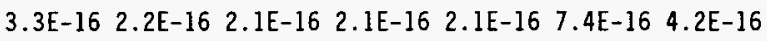
$\begin{array}{llllllll}0.0 E+00 & 0.0 E+00 & 0.0 E+00 & 0.0 E+00 & 0.0 E+00 & 0.0 E+00 & 0.0 E+00\end{array}$ 3.3E-02 1.4E-02 3.1E-04 3.3E-04 3.1E-04 3.7E-04 3.9E-04 $\begin{array}{lllllll}0.0 E+00 & 0.0 E+00 & 0.0 E+00 & 0.0 E+00 & 0.0 E+00 & 0.0 E+00 & 0.0 E+00\end{array}$ 9.5E-01 4.1E-01 2.1E-02 2.3E-02 2.0E-02 3.6E-02 2.9E-02 7.7E-05 1.3E-05 1.6E-05 4.9E-05 1.4E-04 3.4E-04 2.2E-04

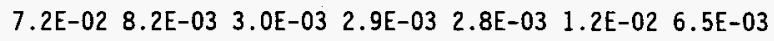
$\begin{array}{llllllll}0.0 E+00 & 0.0 E+00 & 0.0 E+00 & 0.0 E+00 & 0.0 E+00 & 0.0 E+00 & 0.0 E+00\end{array}$ $\begin{array}{lllllll}3.3 \mathrm{E}+00 & 1.2 \mathrm{E}+00 & 1.5 \mathrm{E}-01 & 1.5 \mathrm{E}-01 & 1.4 \mathrm{E}-01 & 1.3 \mathrm{E}-01 & 1.5 \mathrm{E}-01\end{array}$

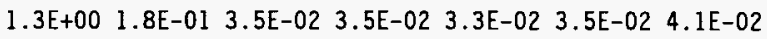

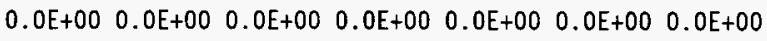
$\begin{array}{lllllll}0.0 E+00 & 0.0 E+00 & 0.0 E+00 & 0.0 E+00 & 0.0 E+00 & 0.0 E+00 & 0.0 E+00\end{array}$

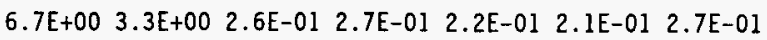


WHC-SD-WM-CN-067, Rev. 0

I 135

7.1E-02

XE 135M

$0.0 \mathrm{E}+00$

$X E 135$

$0.0 \mathrm{E}+00$

CS 135

7. $2 \mathrm{E}-12$

$X E 137$

$0.0 E+00$

CS 137

4. $6 \mathrm{E}-04$

$X E 138$

$0.0 E+00$

CS 138

1.7E-02

Total

4. $4 \mathrm{E}-01$
2. $0 \mathrm{E}+00 \quad 4.4 \mathrm{E}-01 \quad 8.9 \mathrm{E}-02 \quad 8.4 \mathrm{E}-02 \quad 8.0 \mathrm{E}-02 \quad 7.5 \mathrm{E}-02 \quad 9.3 \mathrm{E}-02$

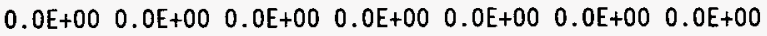

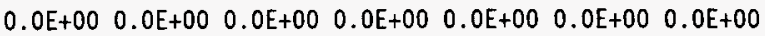

8.4E-12 7.2E-12 7.2E-12 7.2E-12 7.2E-12 8.5E-12 8.9E-12

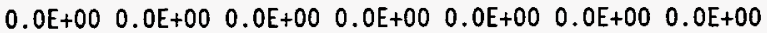

4.6E-04 4.5E-04 4.7E-04 4.6E-04 4.7E-04 4.2E-04 4.4E-04

$\begin{array}{lllllll}0.0 E+00 & 0.0 E+00 & 0.0 E+00 & 0.0 E+00 & 0.0 E+00 & 0.0 E+00 & 0.0 E+00\end{array}$

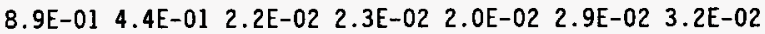

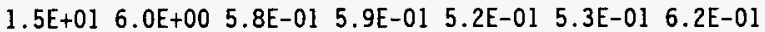


WHC-SD-WM-CN-067, Rev. 0

GENII Dose Calculation Program

(Version 1.485 3-Dec-90)

Case title: WASTE TANK CRITICALITY - ONSITE, Inhalation \& Submersion.

Executed on: $08 / 13 / 96$ at $15: 02: 03$

Page $C$. 5

Acute release

Uptake/exposure period:

Dose commitment period:

Dose units:

Rem $\begin{array}{r}1.0 \\ 50.0\end{array}$

Committed Dose Equivalent by Radionuclide

\begin{tabular}{|c|c|c|c|}
\hline Radionuclide & Ovaries & Muscle & Thyroid \\
\hline KR $83 M$ & $0.0 \mathrm{E}+00$ & $0.0 E+00$ & $0.0 E+0$ \\
\hline KR $85 M$ & $0.0 \mathrm{E}+00$ & $0.0 E+00$ & 0 . \\
\hline KR 85 & $0.0 \mathrm{E}+00$ & $0.0 E+00$ & $0.0 E+00$ \\
\hline KR 87 & $0.0 \mathrm{E}+00$ & $0.0 \mathrm{E}+00$ & $0.0 \mathrm{E}+00$ \\
\hline RB 87 & 16 & -16 & 2. \\
\hline KR 88 & $0.0 \mathrm{E}+00$ & $0.0 \mathrm{E}+00$ & 0.0 \\
\hline RB 88 & 04 & -04 & 3. \\
\hline KR 89 & $0.0 E+00$ & $0.0 E+00$ & $0.0 \mathrm{E}+00$ \\
\hline RB 89 & 1.9 & $2.4 \mathrm{E}-02$ & $2.3 \mathrm{E}-02$ \\
\hline SR 89 & 8. & 8. $5 E-06$ & 8.5 \\
\hline I 131 & 2. & -03 & 3.0 \\
\hline$X E \quad 131 M$ & $0.0 \mathrm{E}+00$ & $0.0 E+00$ & $0.0 \mathrm{E}+00$ \\
\hline I 132 & 1. & $1.7 \mathrm{E}-01$ & 1. \\
\hline I 133 & 3. & $4.8 \mathrm{E}-02$ & $7.6 \mathrm{E}+0$ \\
\hline XE $133 \mathrm{M}$ & 0.0 & $0.0 E+00$ & 0. \\
\hline XE 133 & 0. & $0.0 E+00$ & 0. \\
\hline I 134 & 2. & $E-01$ & +0 \\
\hline I 135 & 7. & 01 & 3. \\
\hline$X E \quad 135 M$ & 0. & .00 & 0. \\
\hline$X E \quad 135$ & & 00 & 0 \\
\hline $\mathrm{CS}$ & & 12 & 7 \\
\hline$X E$ & & 00 & 0. \\
\hline CS 1 & & & \\
\hline XE 138 & & $E+00$ & $=00$ \\
\hline $\operatorname{CS~} 138$ & & & 0 \\
\hline Total & 4.6 & & \\
\hline & 4.0 & & \\
\hline
\end{tabular}




\section{GENII Dose Calculation Program}

(Version 1.485 3-Dec-90)

Case title: WASTE TANK CRITICALITY - ONSITE, Inhalation \& Submersion.

Executed on: $08 / 13 / 96$ at $15: 02: 03$

Page $C$. 6

Acute release

Uptake/exposure period:

Dose commitment period:

Dose units:

\begin{tabular}{|c|c|}
\hline $\begin{array}{l}\text { Radio- } \\
\text { nuclide }\end{array}$ & $\begin{array}{l}\text { Inhalation } \\
\text { Effective } \\
\text { Dose } \\
\text { Equivalent }\end{array}$ \\
\hline $\begin{array}{ll}\text { KR } & 83 M \\
\text { KR } & 85 M \\
\text { KR } & 85 \\
\text { KR } & 87 \\
\text { RB } & 87 \\
\text { KR } & 88 \\
\text { RB } & 88 \\
\text { KR } & 89 \\
\text { RB } & 89 \\
\text { SR } & 89 \\
\text { I } & 131 \\
\text { XE } & 131 M \\
\text { I } & 132 \\
\text { I } & 133 \\
\text { XE } & 133 M \\
X E & 133 \\
\text { I } & 134 \\
\text { I } & 135 \\
\text { XE } & 135 M \\
\text { XE } & 135 \\
C S & 135 \\
\text { XE } & 137 \\
C S & 137 \\
X E & 138 \\
C S & 138\end{array}$ & $\begin{array}{l}0.0 \mathrm{E}+00 \\
0.0 \mathrm{E}+00 \\
0.0 \mathrm{E}+00 \\
0.0 \mathrm{E}+00 \\
2.5 \mathrm{E}-16 \\
0.0 \mathrm{E}+00 \\
5.0 \mathrm{E}-03 \\
0.0 \mathrm{E}+00 \\
1.6 \mathrm{E}-01 \\
6.2 \mathrm{E}-05 \\
9.1 \mathrm{E}-01 \\
0.0 \mathrm{E}+00 \\
1.1 \mathrm{E}+00 \\
2.5 \mathrm{E}+00 \\
0.0 \mathrm{E}+00 \\
0.0 \mathrm{E}+00 \\
1.5 \mathrm{E}+00 \\
1.4 \mathrm{E}+00 \\
0.0 \mathrm{E}+00 \\
0.0 \mathrm{E}+00 \\
7.2 \mathrm{E}-12 \\
0.0 \mathrm{E}+00 \\
4.2 \mathrm{E}-04 \\
0.0 \mathrm{E}+00 \\
1.5 \mathrm{E}-01\end{array}$ \\
\hline
\end{tabular}

Ingestion Effective Dose Equivalent

$0.0 \mathrm{E}+00$

$0.0 \mathrm{E}+00$

$0.0 \mathrm{E}+00$

$0.0 \mathrm{E}+00$

$0.0 \mathrm{E}+00$

$0.0 \mathrm{E}+00$

$0.0 \mathrm{E}+00$

$0.0 \mathrm{E}+00$

$0.0 \mathrm{E}+00$

$0.0 \mathrm{E}+00$

$0.0 \mathrm{E}+00$

$0.0 \mathrm{E}+00$

$0.0 \mathrm{E}+00$

$0.0 \mathrm{E}+00$

$0.0 E+00$

$0.0 \mathrm{E}+00$

$0.0 \mathrm{E}+00$

$0.0 \mathrm{E}+00$

$0.0 \mathrm{E}+00$

$0.0 \mathrm{E}+00$

$0.0 E+00$

$0.0 E+00$

$0.0 E+00$

$0.0 \mathrm{E}+00$

$0.0 E+00$
1.0

50.0

Rem

\begin{tabular}{|c|c|c|}
\hline $\begin{array}{c}\text { External } \\
\text { Dose }\end{array}$ & $\begin{array}{l}\text { Internal } \\
\text { Effective } \\
\text { Dose } \\
\text { Equivalent }\end{array}$ & $\begin{array}{c}\text { Annual } \\
\text { Effective } \\
\text { Dose } \\
\text { Equivalent }\end{array}$ \\
\hline $\begin{array}{l}3.0 \mathrm{E}-05 \\
1.2 \mathrm{E}-03 \\
2.1 \mathrm{E}-10 \\
3.5 \mathrm{E}-02 \\
0.0 \mathrm{E}+00 \\
4.4 \mathrm{E}-02 \\
3.7 \mathrm{E}-04 \\
2.2 \mathrm{E}+00 \\
7.4 \mathrm{E}-02 \\
1.7 \mathrm{E}-11 \\
1.2 \mathrm{E}-04 \\
2.2 \mathrm{E}-07 \\
7.8 \mathrm{E}-02 \\
2.8 \mathrm{E}-03 \\
9.8 \mathrm{E}-06 \\
1.3 \mathrm{E}-04 \\
3.2 \mathrm{E}-01 \\
1.9 \mathrm{E}-02 \\
1.6 \mathrm{E}-01 \\
1.1 \mathrm{E}-02 \\
0.0 \mathrm{E}+00 \\
9.1 \mathrm{E}-01 \\
8.2 \mathrm{E}-08 \\
1.2 \mathrm{E}+00 \\
3.7 \mathrm{E}-02\end{array}$ & $\begin{array}{l}0.0 \mathrm{E}+00 \\
0.0 \mathrm{E}+00 \\
0.0 \mathrm{E}+00 \\
0.0 \mathrm{E}+00 \\
2.5 \mathrm{E}-16 \\
0.0 \mathrm{E}+00 \\
5.0 \mathrm{E}-03 \\
0.0 \mathrm{E}+00 \\
1.6 \mathrm{E}-01 \\
6.2 \mathrm{E}-05 \\
9.1 \mathrm{E}-01 \\
0.0 \mathrm{E}+00 \\
1.1 \mathrm{E}+00 \\
2.5 \mathrm{E}+00 \\
0.0 \mathrm{E}+00 \\
0.0 \mathrm{E}+00 \\
1.5 \mathrm{E}+00 \\
1.4 \mathrm{E}+00 \\
0.0 \mathrm{E}+00 \\
0.0 \mathrm{E}+00 \\
7.2 \mathrm{E}-12 \\
0.0 \mathrm{E}+00 \\
4.2 \mathrm{E}-04 \\
0.0 \mathrm{E}+00 \\
1.5 \mathrm{E}-01\end{array}$ & $\begin{array}{l}3.0 \mathrm{E}-05 \\
1.2 \mathrm{E}-03 \\
2.1 \mathrm{E}-10 \\
3.5 \mathrm{E}-02 \\
2.5 \mathrm{E}-16 \\
4.4 \mathrm{E}-02 \\
5.4 \mathrm{E}-03 \\
2.2 \mathrm{E}+00 \\
2.3 \mathrm{E}-01 \\
6.2 \mathrm{E}-05 \\
9.1 \mathrm{E}-01 \\
2.2 \mathrm{E}-07 \\
1.2 \mathrm{E}+00 \\
2.5 \mathrm{E}+00 \\
9.8 \mathrm{E}-06 \\
1.3 \mathrm{E}-04 \\
1.9 \mathrm{E}+00 \\
1.4 \mathrm{E}+00 \\
1.6 \mathrm{E}-01 \\
1.1 \mathrm{E}-02 \\
7.2 \mathrm{E}-12 \\
9.1 \mathrm{E}-01 \\
4.2 \mathrm{E}-04 \\
1.2 \mathrm{E}+00 \\
1.9 \mathrm{E}-01\end{array}$ \\
\hline
\end{tabular}


WHC-SD-WM-CN-057, Rev, 0

\section{GENII Dose Calculation Program}

(Version 1.485 3-Dec-90)

Case title: WASTE TANK CRITICALITY - OFFSITE, Inhalation \& Submersion.

Executed on: $08 / 13 / 96$ at 15:06:32

Page A.

This is a far-field (wide-scale release, multiple site) scenario.

Release is acute

Individual dose

THE FOLLOWING TRANSPORT MODES ARE CONSIDERED

Air

THE FOLLOWING EXPOSURE PATHS ARE CONSIDERED:

Finite plume, external

Inhalation uptake

THE FOLLOWING TIMES ARE USED:

Intake ends after $(y r)$ : $\quad 1.0$

Dose calculations ends after $(y r): \quad 50.0$

$==x==x==x=$ FILENAMES AND TITLES OF FILES/LIBRARIES USED

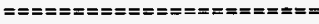

Input file name: IGENII\TANKFfC2.IN

GENII Default Parameter Values (28-Mar-90 RAP)

Radionuclide Library - Times $<100$ years (23-July-93 PDR)

External Dose Factors for GENII in person $5 \mathrm{v} / \mathrm{yr}$ per Bq/n (8-May-90 R

Worst-Case Solubilities, Yearly Dose Increments (23-Jul-93 PDR)

EXTGAM - Gamma Energies by Group for Finite Plume (13-May-90 RAP)

200 AREA (HMS) - 10 M - Pasqui11 A - G (1983 - 1991 Average)

\begin{tabular}{|c|c|c|c|}
\hline $\begin{array}{l}\text { Release } \\
\text { Radio- } \\
\text { nuclide }\end{array}$ & $\underset{\mathrm{Ci} / \mathrm{yr}}{\operatorname{Air}}$ & $\begin{array}{c}\text { Surface } \\
\text { Water } \\
\text { Ci/yr }\end{array}$ & $\begin{array}{l}\text { Buried } \\
\text { Source } \\
\mathrm{Ci} / \mathrm{m} 3\end{array}$ \\
\hline $\begin{array}{l}\text { KR83M } \\
\text { KR85M } \\
\text { KR85 } \\
\text { KR87 } \\
\text { KR88 } \\
\text { KR89 }\end{array}$ & $\begin{array}{l}1.1 \mathrm{E}+02 \\
7.1 \mathrm{E}+01 \\
8.1 \mathrm{E}-04 \\
4.3 \mathrm{E}+02 \\
2.3 \mathrm{E}+02 \\
1.3 \mathrm{E}+04\end{array}$ & $\begin{array}{l}0.0 \mathrm{E}+00 \\
0.0 \mathrm{E}+00 \\
0.0 \mathrm{E}+00 \\
0.0 \mathrm{E}+00 \\
0.0 \mathrm{E}+00 \\
0.0 \mathrm{E}+00\end{array}$ & $\begin{array}{l}0.0 \mathrm{E}+00 \\
0.0 \mathrm{E}+00 \\
0.0 \mathrm{E}+00 \\
0.0 \mathrm{E}+00 \\
0.0 \mathrm{E}+00 \\
0.0 \mathrm{E}+00\end{array}$ \\
\hline
\end{tabular}


WHC-SD-WM-CN-067, Rev. 0

$\begin{array}{lllll}X E 131 M & 1.0 E-01 & 0.0 E+00 & 0.0 E+00\end{array}$

$X E 133 \mathrm{M} \quad 2.2 \mathrm{E}+00 \quad 0.0 \mathrm{E}+00 \quad 0.0 \mathrm{E}+00$

$\begin{array}{lllll}X E 133 & 2.7 E+01 & 0.0 E+00 & 0.0 E+00\end{array}$

$\begin{array}{lllll}X E 135 M & 3.3 \mathrm{E}+03 & 0.0 \mathrm{E}+00 & 0.0 \mathrm{E}+00\end{array}$

$\begin{array}{llll}X E 135 & 4.1 \mathrm{E}+02 & 0.0 \mathrm{E}+00 & 0.0 \mathrm{E}+00\end{array}$

XE137 4.9E +04 $0.0 \mathrm{E}+00 \quad 0.0 \mathrm{E}+00$

$X E 138 \quad 1.1 \mathrm{E}+04 \quad 0.0 \mathrm{E}+00 \quad 0.0 \mathrm{E}+00$

I $131 \quad 2.8 \mathrm{E}+00 \quad 0.0 \mathrm{E}+00 \quad 0.0 \mathrm{E}+00$

I $1323.0 \mathrm{E}+02 \quad 0.0 \mathrm{E}+00 \quad 0.0 \mathrm{E}+00$

$\begin{array}{lllll}133 & 4.0 \mathrm{E}+01 & 0.0 \mathrm{E}+00 & 0.0 \mathrm{E}+00\end{array}$

$\begin{array}{lllll}134 & 1.1 \mathrm{E}+03 & 0.0 \mathrm{E}+00 & 0.0 \mathrm{E}+00\end{array}$

I $135 \quad 1.1 \mathrm{E}+02 \quad 0.0 \mathrm{E}+00 \quad 0.0 \mathrm{E}+00$

$====x====$ AIR TRANSPORT

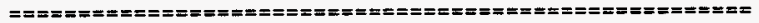

Joint frequency data input.

8.8E+03 Maximum individual distance from release point (m)

$9.0 \mathrm{E}+00$ Maximum individual sector index (Wind Toward $\mathrm{N}$ )

Ground level release.

$==s=n=s=s$ EXTERNAL EXPOSURE

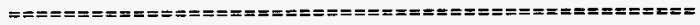

$1.0 \mathrm{E}+00$ Fraction of time spent in cloud

$==x==x==x=$ INHALATION

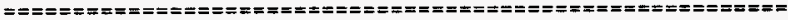

Resuspension not considered

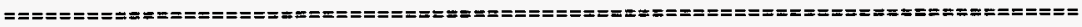

$=$

Input prepared by:

Date:

Input checked by:

Date: 
WHC-SD-WM-CN-067, Rev. 0

\section{GENII Dose Calculation Program}

(Version 1.485 3-Dec-90)

Case title: WASTE TANK CRITICALITY - OFFSITE, Inhalation \& Submersion.

Executed on: 08/13/96 at 15:06:32

Page A. 2

Sector Index: 9

Probability: $5.0 \mathrm{E}-02$

\begin{tabular}{|c|c|c|c|c|c|c|c|}
\hline is & $\begin{array}{l}\text { Travel } \\
\text { Time } \\
\text { (sec) }\end{array}$ & $\begin{array}{l}\text { Energy } \\
0.15 \\
(\mathrm{MeV})\end{array}$ & $\begin{array}{l}\text { Energy } \\
0.4 \\
(\mathrm{MeV})\end{array}$ & $\begin{array}{l}\text { Energy } \\
0.75 \\
(\mathrm{MeV})\end{array}$ & $\begin{array}{l}\text { Energy } \\
1.25 \\
(\mathrm{MeV})\end{array}$ & $\begin{array}{l}\text { Energy } \\
1.75 \\
(\mathrm{MeV})\end{array}$ & $\begin{array}{l}\text { Energy } \\
2.25 \\
(\mathrm{MeV})\end{array}$ \\
\hline & & . & -06 & -06 & $5.0 \mathrm{E}-0$ & $E-c$ & \\
\hline
\end{tabular}


WHC-SD-WM-CN-067, Rev. 0

GENII Dose Calculation Program

(Version 1.485 3-Dec-90)

Case title: WASTE TANK CRITICALITY - OFFSITE, Inhalation \& Submersion.

Executed on: $08 / 13 / 96$ at $15: 06: 40$

Page B.

1

5.4E-05 Individual $\mathrm{E} / \mathrm{Q}$ 


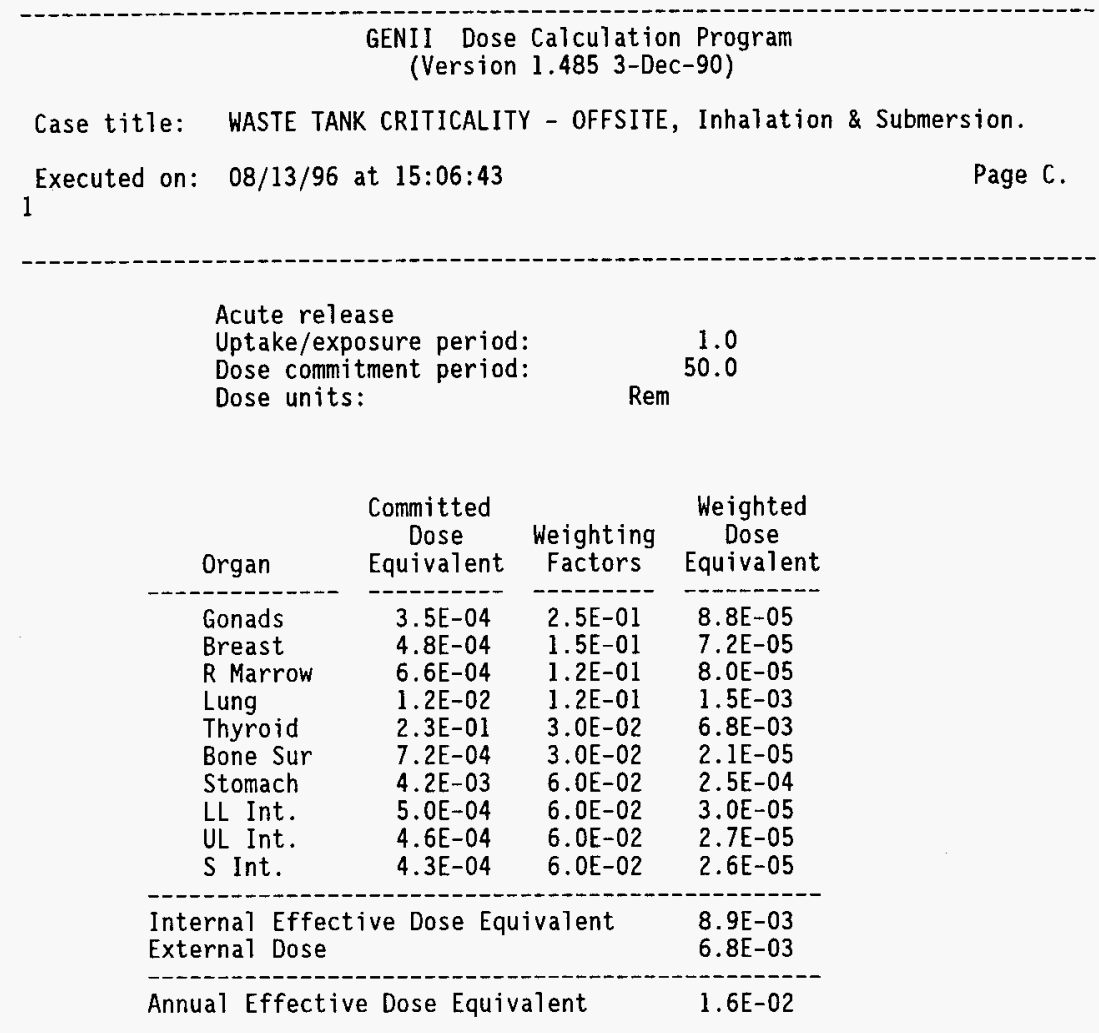

\begin{tabular}{ll} 
Controlling Organ: & Thyroid \\
Controlling Pathway: & Inh \\
Controlling Radionuclide: & I 133 \\
\hline Total Inhalation EDE: & $8.9 \mathrm{E}-03$ \\
Total Ingestion EDE: & $0.0 \mathrm{E}+00$ \\
\hline
\end{tabular}


WHC-SD-WH-CN-067, Rev. O

GENII Dose Calculation Program

(Version 1.485 3-Dec-90)

Case titie: WASTE TANK CRITICALITY - OFFSITE, Inhalation \& Submersion.

Executed on: $08 / 13 / 96$ at $15: 06: 43$

Page $C$. 2

Acute release

Uptake/exposure period:

Dose commitment period:

Dose units:
Dose Commitment Year

$\begin{array}{llll}1 & 2 & 3 & \ldots\end{array}$

\section{Internal : |}

Intake : I

Year: 3 i

$$
0.0 E+00 \quad \ldots
$$

i

2 i

$$
0.0 \mathrm{E}+00
$$

$0.0 E+00$

$\ldots$

Internal

I

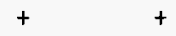

Effective

$1: 8.8$

$$
\begin{gathered}
8.8 E-03+1.0 E-06+5.7 E-08 \\
\text { i }
\end{gathered}
$$

Internal

Annua 1

$$
8.8 \mathrm{E}-03
$$$$
+1.0 \mathrm{E}-06+
$$$$
+5.7 E-08+\ldots
$$$$
=
$$

8. $9 E-03$

Dose

$$
+
$$

External

Annua 1

$6.8 \mathrm{E}-03$

$0.0 \mathrm{E}+00$

$0.0 E+00$

$\ldots$

$6.8 \mathrm{E}-03$

Dose 
WHC-SD-WM-CN-067, Rev. 0

in $\quad$ i $\quad$ H

Annual

Dose

$1.6 \mathrm{E}-02+1.0 \mathrm{E}-06+5.7 \mathrm{E}-08+\ldots$

$=1.6 \mathrm{E}-02$

Cumulative

Maximum

1.6E-02 Annua 1

Dose Occurred

In Year 1 


\section{GENII Dose Calculation Program}

(Version 1.485 3-Dec-90)

Case title: WASTE TANK CRITICALITY - OFFSITE, Inhalation \& Submersion. 3

Executed on: $08 / 13 / 96$ at $15: 06: 43$

Page C.

Acute release

Uptake/exposure period:

Dose commitment period:

Dose units:

$\operatorname{Rem} \begin{array}{r}1.0 \\ 50.0\end{array}$

Committed Dose Equivalent by Exposure Pathway

Pathway Lung Stomach S Int. UL Int. LL Int. Bone Su R Marro Testes

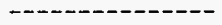

Inhale

$3.4 \mathrm{E}-04$

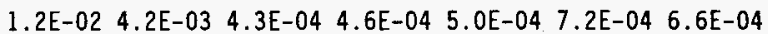

-------------

Total

1.2E-02 4.2E-03 4.3E-04 4.6E-04 5.0E-04 7.2E-04 6.6E-04

3. $4 \mathrm{E}-04$

\begin{tabular}{llll} 
Pathway & Ovaries Muscle & Thyroid \\
\hline Inhale & $3.5 \mathrm{E}-04$ & $4.8 \mathrm{E}-04$ & $2.3 \mathrm{E}-01$ \\
\hline Total & $3.5 \mathrm{E}-04$ & $4.8 \mathrm{E}-04$ & $2.3 \mathrm{E}-01$
\end{tabular}

External Dose by Exposure Pathway

Pathway

\begin{tabular}{ll} 
Plume & - \\
\hline Total & $6.8 \mathrm{E}-03$ \\
\hline$-8.8 \mathrm{E}-03$
\end{tabular}


(Version 1.485 3-Dec-90)

Case title: WASTE TANK CRITICALITY - OFFSITE, Inhalation \& Submersion. 4 Executed on: $08 / 13 / 96$ at 15:06:43

Page $C$.

Acute release

Uptake/exposure period: Dose commitment period: Dose units:
1.0
50.0
Rem

Committed Dose Equivalent by Radionuclide

Radionuclide Lung Stomach S Int. UL Int. LL Int. Bone Su R Marro Testes
KR 83M
$0.0 \mathrm{E}+00$

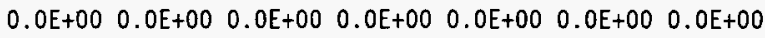
KR $85 \mathrm{M}$
$\begin{array}{lllllll}0.0 E+00 & 0.0 E+00 & 0.0 E+00 & 0.0 E+00 & 0.0 E+00 & 0.0 E+00 & 0.0 E+00\end{array}$
$0.0 \mathrm{E}+00$
$\begin{array}{llllllll}0.0 E+00 & 0.0 E+00 & 0.0 E+00 & 0.0 E+00 & 0.0 E+00 & 0.0 E+00 & 0.0 E+00\end{array}$
$0.0 E+00$
$\begin{array}{llllllll}0.0 E+00 & 0.0 E+00 & 0.0 E+00 & 0.0 E+00 & 0.0 E+00 & 0.0 E+00 & 0.0 E+00\end{array}$
KR 87
$0.0 \mathrm{E}+00$
$\mathrm{RB} 87$
$4.8 \mathrm{E}-17$
KR 88
$0.0 E+00$
RB 88
1. $1 \mathrm{E}-05$
KR 89
$0.0 \mathrm{E}+00$
RB 89
$1.8 \mathrm{E}-07$
SR 89
7.8E-06
I 131
4. $0 \mathrm{E}-06$
XE $131 M$
$0.0 \mathrm{E}+00$

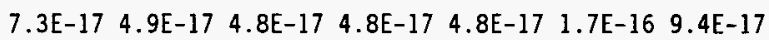
$\begin{array}{lllllll}0.0 E+00 & 0.0 E+00 & 0.0 E+00 & 0.0 E+00 & 0.0 E+00 & 0.0 E+00 & 0.0 E+00\end{array}$
1.3E-03 5.4E-04 1.2E-05 1.3E-05 1.2E-05 1.4E-05 1.5E-05
$\begin{array}{lllllll}0.0 E+00 & 0.0 E+00 & 0.0 E+00 & 0.0 E+00 & 0.0 E+00 & 0.0 E+00 & 0.0 E+00\end{array}$
$\begin{array}{lllllll}1.0 \mathrm{E}-05 & 4.4 \mathrm{E}-06 & 2.2 \mathrm{E}-07 & 2.4 \mathrm{E}-07 & 2.1 \mathrm{E}-0 & 3.8 \mathrm{E}-07 & 3.1 \mathrm{E}-07\end{array}$

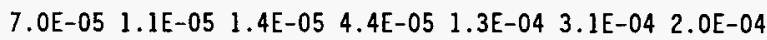
1.2E-04 1.3E-05 4.9E-06 4.7E-06 4.5E-06 2.0E-05 1.1E-05
$\begin{array}{llllllll}0.0 E+00 & 0.0 E+00 & 0.0 E+00 & 0.0 E+00 & 0.0 E+00 & 0.0 E+00 & 0.0 E+00\end{array}$
I 132
8. $5 E-05$
I 133
$4.8 \mathrm{E}-05$
$X E 133 M$
$0.0 \mathrm{E}+00$
$X E 133$
$0.0 E+00$
I 134
3.6E-05
2.4E-03 8.5E-04 1.1E-04 1.1E-04 1.0E-04 9.4E-05 1.1E-04
2.0E-03 2.6E-04 5.3E-05 5.3E-05 5.1E-05 5.3E-05 6.3E-05
$\begin{array}{llllllll}0.0 E+00 & 0.0 E+00 & 0.0 E+00 & 0.0 E+00 & 0.0 E+00 & 0.0 E+00 & 0.0 E+00\end{array}$
$\begin{array}{lllllll}0.0 E+00 & 0.0 E+00 & 0.0 E+00 & 0.0 E+00 & 0.0 E+00 & 0.0 E+00 & 0.0 E+00\end{array}$

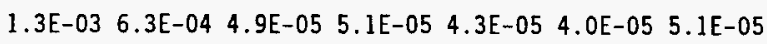


WHC-SD-WM-CN-067, Rev. 0

I 135

8. $9 E-05$ XE $135 \mathrm{M}$

$0.0 E+00$ $X E 135$

$0.0 E+00$

CS 135

3. $4 \mathrm{E}-12$

$X E 137$

$0.0 \mathrm{E}+00$

CS 137

6. $9 E-06$

XE 138

$0.0 E+00$

CS 138

4. $9 E-05$

Total

3. $4 \mathrm{E}-04$
$2.6 \mathrm{E}-03 \quad 5.6 \mathrm{E}-04 \quad 1.1 \mathrm{E}-04 \quad 1.1 \mathrm{E}-04 \quad 1.0 \mathrm{E}-04 \quad 9.4 \mathrm{E}-05 \quad 1.2 \mathrm{E}-04$

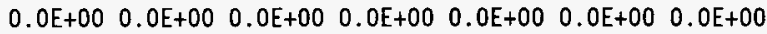

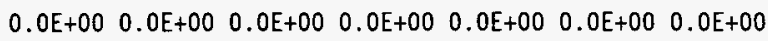

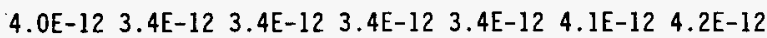

$\begin{array}{lllllll}0.0 E+00 & 0.0 E+00 & 0.0 E+00 & 0.0 E+00 & 0.0 E+00 & 0.0 E+00 & 0.0 E+00\end{array}$

6.9E-06 6.8E-06 7.1E-06 7.0E-06 7.1E-06 6.3E-06 6.5E-06

$\begin{array}{lllllll}0.0 E+00 & 0.0 E+00 & 0.0 E+00 & 0.0 E+00 & 0.0 E+00 & 0.0 E+00 & 0.0 E+00\end{array}$

2.6E-03 1.3E-03 6.3E-05 6.7E-05 5.9E-05 8.5E-05 9.3E-05

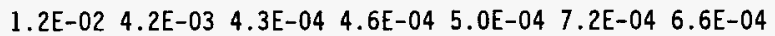


WHC-SD-WM-CN-067, Rev, 0

\section{GENII Dose Calculation Program}

(Version $1.4853-$ Dec-90)

Case title: WASTE TANK CRITICALITY - OFFSITE, Inhalation \& Submersion.

Executed on: 08/13/96 at 15:06:43 Page C. 5

Acute release

Uptake/exposure period:

Dose commitment period:

Dose units:

1.0

Rem

50.0

Committed Dose Equivalent by Radionuclide

Radionuclide

KR 83M

KR $85 M$

KR 85

KR 87

RB 87

KR 88

RB 88

KR 89

RB 89

SR 89

I 131

XE $131 \mathrm{M}$

I 132

I 133

XE $133 \mathrm{M}$

$X E 133$

I 134

I 135

XE 135M

XE 135

CS 135

XE 137

CS 137

XE 138

CS 138

Total
Ovaries Muscle Thyroid

$0.0 \mathrm{E}+000.0 \mathrm{E}+00 \quad 0.0 \mathrm{E}+00$

$0.0 \mathrm{E}+00 \quad 0.0 \mathrm{E}+00 \quad 0.0 \mathrm{E}+00$

$0.0 E+00 \quad 0.0 E+00 \quad 0.0 E+00$

$0.0 \mathrm{E}+00 \quad 0.0 \mathrm{E}+00 \quad 0.0 \mathrm{E}+00$

4.8E-17 4.8E-17 4.8E-17

$0.0 E+00 \quad 0.0 E+00 \quad 0.0 E+00$

1.2E-05 1.3E-05 1.2E-05

$0.0 \mathrm{E}+00 \quad 0.0 \mathrm{E}+00 \quad 0.0 \mathrm{E}+00$

2.0E-07 2.5E-07 2.4E-07

$7.8 \mathrm{E}-06 \quad 7.8 \mathrm{E}-067.8 \mathrm{E}-06$

4.4E-06 1.4E-05 4.9E-02

$0.0 \mathrm{E}+00 \quad 0.0 \mathrm{E}+00 \quad 0.0 \mathrm{E}+00$

8.5E-05 1.2E-04 1.4E-02

4.8E-05 7.2E-05 1.2E-01

$0.0 \mathrm{E}+00 \quad 0.0 \mathrm{E}+00 \quad 0.0 \mathrm{E}+00$

$0.0 \mathrm{E}+00 \quad 0.0 \mathrm{E}+00 \quad 0.0 \mathrm{E}+00$

3.7E-05 5.3E-05 2.4E-03

9.4E-05 1.3E-04 4.6E-02

$0.0 \mathrm{E}+00 \quad 0.0 \mathrm{E}+00 \quad 0.0 \mathrm{E}+00$

$0.0 \mathrm{E}+00 \quad 0.0 \mathrm{E}+00 \quad 0.0 \mathrm{E}+00$

3. $4 \mathrm{E}-12$ 3. $4 \mathrm{E}-12$ 3. $4 \mathrm{E}-12$

$0.0 \mathrm{E}+00 \quad 0.0 \mathrm{E}+00 \quad 0.0 \mathrm{E}+00$

6.4E-06 6.2E-06 6.2E-06

$0.0 \mathrm{E}+00 \quad 0.0 \mathrm{E}+00 \quad 0.0 \mathrm{E}+00$

5.7E-05 7.0E-05 6.2E-05

3. $5 \mathrm{E}-04 \quad 4.8 \mathrm{E}-04 \quad 2.3 \mathrm{E}-01$ 


\section{WHC-SD-WM-CN-067, Rev. 0}

\section{GENII Dose Calculation Program}

(Version 1.485 3-Dec-90)

Case title: WASTE TANK CRITICALITY - OFFSITE, Inhalation \& submersion. 6

Executed on: $08 / 13 / 96$ at $15: 06: 43$

Page $C$.

Acute release

Uptake/exposure period:

Dose commitment period:

Dose units:

Inhalation Ingestion

Effective Effective

Radio-

nuclide

-.------

KR $83 \mathrm{M}$

KR $85 M$

KR 85

KR 87

RB 87

KR 88

RB 88

KR 89

RB 89

SR 89

I 131

XE 13IM

I 132

I 133

XE 133M

$X E 133$

I 134

I 135

XE $135 \mathrm{M}$

XE 135

CS 135

XE 137

CS 137

XE 138

CS 138
Dose

Equivalent

$0.0 \mathrm{E}+00$

$0.0 \mathrm{E}+00$

$0.0 \mathrm{E}+00$

$0.0 \mathrm{E}+00$

5.7E-17

$0.0 E+00$

2. $0 \mathrm{E}-04$

$0.0 E+00$

1. $6 \mathrm{E}-06$

5. $6 \mathrm{E}-05$

1. $5 E-03$

$0.0 E+00$

8. $2 \mathrm{E}-04$

3. $8 \mathrm{E}-03$

$0.0 E+00$

$0.0 E+00$

3. $0 \mathrm{E}-04$

$1.8 \mathrm{E}-03$

$0.0 \mathrm{E}+00$

$0.0 \mathrm{E}+00$

3. $4 \mathrm{E}-12$

$0.0 E+00$

6. 3E-06

$0.0 \mathrm{E}+00$

4. $4 \mathrm{E}-04$
Dose

Equivalent

$0.0 \mathrm{E}+00$

$0.0 \mathrm{E}+00$

$0.0 E+00$

$0.0 E+00$

$0.0 E+00$

$0.0 \mathrm{E}+00$

$0.0 E+00$

$0.0 E+00$

$0.0 E+00$

$0.0 E+00$

$0.0 \mathrm{E}+00$

$0.0 E+00$

$0.0 \mathrm{E}+00$

$0.0 E+00$

$0.0 E+00$

$0.0 \mathrm{E}+00$

$0.0 E+00$

$0.0 E+00$

$0.0 E+00$

$0.0 E+00$

$0.0 E+00$

$0.0 \mathrm{E}+00$

$0.0 E+00$

$0.0 \mathrm{E}+00$

$0.0 E+00$
External Dose

4.3E-07

3. $1 \mathrm{E}-05$

6. $4 \mathrm{E}-12$

2.7E-04

$0.0 \mathrm{E}+00$

7. $7 \mathrm{E}-04$

2. $9 \mathrm{E}-04$

2. $2 \mathrm{E}-17$

1. $5 \mathrm{E}-05$

2. $8 \mathrm{E}-10$

4. 1E-06

8. $6 \mathrm{E}-09$

1. $1 \mathrm{E}-03$

7. $9 \mathrm{E}-05$

3. $8 \mathrm{E}-07$

5. $3 \mathrm{E}-06$

1. $2 \mathrm{E}-03$

4. $6 \mathrm{E}-04$

2. $5 \mathrm{E}-05$

4. 5E-04

$0.0 \mathrm{E}+00$

4. $6 \mathrm{E}-15$

2. $4 \mathrm{E}-08$

1. $4 \mathrm{E}-05$

2.1E-03
1.0

50.0

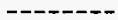

Internal Annual

Effective Effective

Dose Dose

Equivalent Equivalent

$0.0 \mathrm{E}+00$

$0.0 \mathrm{E}+00$

$0.0 \mathrm{E}+00$

$0.0 \mathrm{E}+00$

5. $7 \mathrm{E}-17$

$0.0 \mathrm{E}+00$

2. $0 \mathrm{E}-04$

$0.0 \mathrm{E}+00$

1. $6 \mathrm{E}-06$

5. $6 \mathrm{E}-05$

1. $5 \mathrm{E}-03$

$0.0 \mathrm{E}+00$

8. $2 \mathrm{E}-04$

$3.8 \mathrm{E}-03$

$0.0 \mathrm{E}+00$

$0.0 \mathrm{E}+00$

3. $0 \mathrm{E}-04$

1. $8 \mathrm{E}-03$

$0.0 \mathrm{E}+00$

$0.0 \mathrm{E}+00$

3. $4 \mathrm{E}-12$

$0.0 \mathrm{E}+00$

$6.3 \mathrm{E}-06$

$0.0 \mathrm{E}+00$

4. 4E-04
4. $3 \mathrm{E}-07$

3.1E-05

6. $4 \mathrm{E}-12$

2.7E-04

5.7E-17

7. $7 \mathrm{E}-04$

4. $9 \mathrm{E}-04$

2. $2 \mathrm{E}-17$

$1.7 \mathrm{E}-05$

5. $6 \mathrm{E}-05$

1. $5 \mathrm{E}-03$

8. $6 \mathrm{E}-09$

1. $9 \mathrm{E}-03$

3. $8 \mathrm{E}-03$

3. $8 \mathrm{E}-07$

5. $3 \mathrm{E}-06$

1. $5 \mathrm{E}-03$

2. $2 \mathrm{E}-03$

2. $5 \mathrm{E}-05$

4. $5 \mathrm{E}-04$

3. $4 \mathrm{E}-12$

4. $6 \mathrm{E}-15$

6. $3 \mathrm{E}-06$

1. $4 \mathrm{E}-05$

2. $5 \mathrm{E}-03$ 


\section{CHECKLIST FOR PEER REVIEW}

Document Reviewed: C. H. Huang, "GENII DOSE CALCULATIONS FOR RADIOACTIVE LIQUID WASTE TANK CRITICALITY," August 22, 1996.

Scope of Review: Entire Document

Yes No NA

[ ] [ ] [ $*$ Previous reviews complete and cover analysis, up to scope of this review, with no gaps. Problem completely defined. Accident scenarios developed in a clear and logical manner. Necessary assumptions explicitly stated and supported. Computer codes and data files documented. Data used in calculations explicitly stated in document. Data checked for consistency with original source information as applicable.

[ ] [ ] [ $\$ Mathematical derivations checked including dimensional consistency of results.

W [ ] [ ] Models appropriate and used within range of validity or use outside range of established validity justified.

$\triangle[$ [ ] [ ] Hand calculations checked for errors. Spreadsheet results should be treated exactly the same as hand calculations. [ ] [ ] Software input correct and consistent with document reviewed. $D$ [ ] [ ] Software output consistent with input and with results reported in document reviewed.

[ ] [ ] $\$ Limits/criteria/guidelines applied to analysis results are appropriate and referenced. Limits/criteria/guidelines checked against references.

[ ] [ ] $\bowtie$ Safety margins consistent with good engineering practices. A [ ] [ ] Conclusions consistent with analytical results and applicable limits.

$\triangle[$ [ [ ] Results and conclusions address all points required in the problem statement.

[ ] [ ] DA Format consistent with appropriate NRC Regulatory Guide or other standards

[ ] $\mathcal{A}^{*}$ Review calculations, comments, and/or notes are attached.

[४[ ] [ ]

Document approved

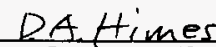

Reviewer (Printed Name and Signature)

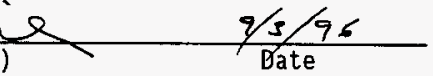


WHC-SD-WM-CN-067, Rev. O

\section{HEDOP REVIEW CHECKLIST \\ for \\ Radiological and Nonradiological Release Calculations}

Document Reviewed: C. H. Huang, "GENII DOSE CALCULATIONS FOR RADIOACTIVE

LIQUID WASTE TANK CRITICALITY," Augsust 22, 1996.

Submitted by: C. H. HUANG

Date Submitted: August 22, 1996

Scope of Review: Entire Document

YES NO* N/A

4 [ ] [ ] 1. A detailed technical review and approval of the environmental transport and dose calculation portion of the analysis has been performed and documented.

[ ] [ ] [ 4 2. Detailed technical review(s) and approval(s) of scenario and release determinations have been performed and documented.

D [ ] [ ] 3. HEDOP-approved code(s) were used.

[] [ ] $]$ 4. Receptor locations were selected according to HEDOP recommendations.

\&] [ ] [ ] 5. All applicable environmental pathways and code options were included and are appropriate for the calculations.

4 [ ] [ ] 6. Hanford site data were used.

B. [ ] [ ] 7. Model adjustments external to the computer program were justified and performed correctly.

(X] [ ] [ ] 8. The analysis is consistent with HEDOP recommendations.

9. Supporting notes, calculations, comments, comment resolutions, or other information is attached. (Use the "Page 1 of $X$ " page numbering format and sign and date each added page.)

[X] [ ] 10. Approval is granted on behalf of the Hanford Environmentai Dose Overview Panel.

* A11 "NO" responses must be explained and use of nonstandard methods justified.

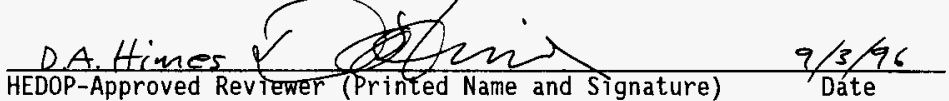

COMMENTS (add additional signed and dated pages if necessary): 


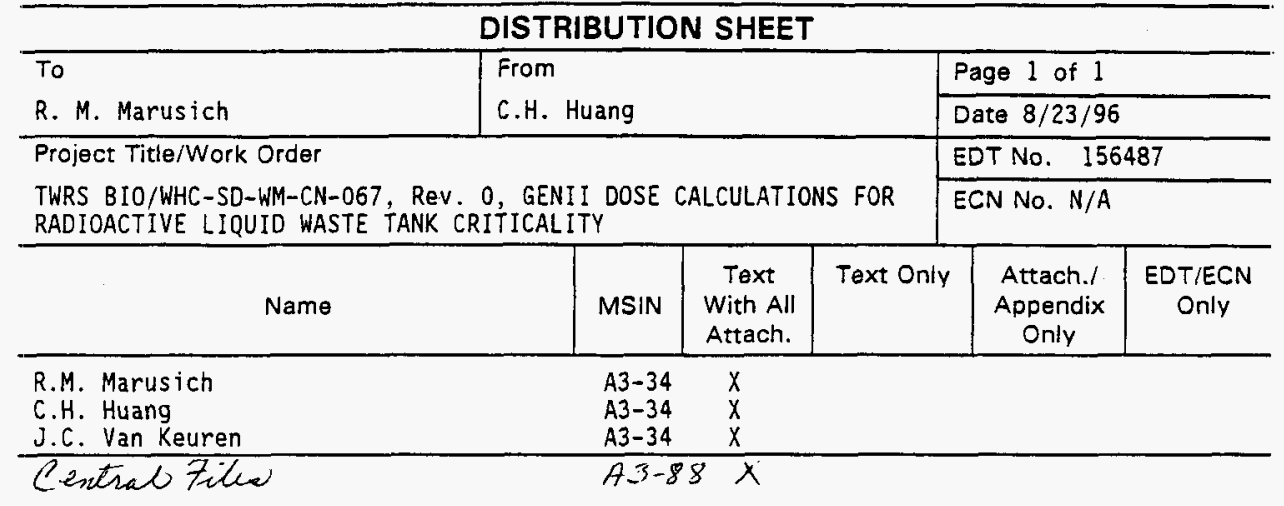

